\title{
On the role of curvature in the elastic energy of non-Euclidean thin bodies
}

\author{
Cy Maor* and Asaf Shachar ${ }^{\dagger}$
}

\begin{abstract}
We prove a relation between the scaling $h^{\beta}$ of the elastic energies of shrinking nonEuclidean bodies $\mathcal{S}_{h}$ of thickness $h \rightarrow 0$, and the curvature along their mid-surface $\mathcal{S}$. This extends and generalizes similar results for plates [BLS16, LRR] to any dimension and co-dimension. In particular, it proves that the natural scaling for non-Euclidean rods with smooth metric is $h^{4}$, as claimed in [AAE ${ }^{+}$] using a formal asymptotic expansion. The proof involves calculating the $\Gamma$-limit for the elastic energies of small balls $B_{h}(p)$, scaled by $h^{4}$, and showing that the limit infimum energy is given by a square of a norm of the curvature at a point $p$. This $\Gamma$-limit proves asymptotics calculated in $\left[\mathrm{AKM}^{+} 16\right]$.
\end{abstract}

\section{Contents}

1 Introduction and main results

1.1 Non-Euclidean elasticity . . . . . . . . . . . . . . . . . . . . . . 2

1.2 Thin elastic bodies . . . . . . . . . . . . . . . 2

1.3 Main results .......................... 4

2 - 2 -limit of the elastic energy of shrinking balls 7

2.1 The energy scaling of the exponential map . . . . . . . . . . 8

2.2 Proof of Theorem $2.1(\Gamma$-convergence $) \ldots \ldots \ldots \ldots$

2.2 .1 Rigidity (part 1a) . . . . . . . . . . . . . . . . 9

2.2.2 Compactness and lower bound (parts $1 \mathrm{~b}$ and $1 \mathrm{c}$ ) . . . . . . . . 10

2.2 .3 Upper bound (part 2) . . . . . . . . . . . . . . 12

2.3 Proof of Theorem 1.1 (limit of infima) . . . . . . . . . . . 13

3 Energy scaling for general thin elastic bodies

3.1 Proofs regarding the scaling inf $E_{\delta_{h}}=o\left(h^{2}\right) \ldots \ldots \ldots \ldots \ldots$

3.2 Proofs regarding the scaling inf $E_{\delta_{h}}=O\left(h^{4}\right) \ldots \ldots \ldots \ldots$

${ }^{*}$ Department of Mathematics, University of Toronto.

${ }^{+}$Institute of Mathematics, The Hebrew University. 


\section{Introduction and main results}

\subsection{Non-Euclidean elasticity}

Non-Euclidean, or incompatible elasticity is an elastic theory for bodies that do not have a reference configuration, i.e. a stress-free configuration (therefore they are commonly referred to as pre-stressed bodies). This theory has numerous applications - it originated in the 1950's in the context of crystalline defects (see e.g. [Kon55, BBS55, BS56]), and in recent years it is motivated by studies of growing tissues, thermal expansion, and other mechanics involving differential expansion or shrinkage $\mathrm{AESK}_{11}, \mathrm{AAE}^{+} 12$, OY09, KES07, GSD16, AKM+16].

Mathematically, a pre-stressed elastic body is modeled as an $n$-dimensional compact, oriented Riemannian manifold $\left(\mathcal{N}^{n}, \mathfrak{g}\right)$. It is "incompatible" if $\mathfrak{g}$ is not flat. Given a configuration $u: \mathcal{N} \rightarrow \mathbb{R}^{n}$, the elastic energy density at a point $p \in \mathcal{M}$ measures the strain - the discrepancy between the intrinsic metric $g$ and the actual metric $u^{\star} e$ ind uced by the configuration ( $e$ being the Euclidean metric in $\mathbb{R}^{n}$ ). A prototypical "Hookean" energy is

$$
E_{\mathcal{M}}: W^{1,2}\left(\mathcal{M} ; \mathbb{R}^{n}\right) \rightarrow \mathbb{R}, \quad E_{\mathcal{M}}[u]:=\int_{\mathcal{M}} \operatorname{dist}^{2}(d u, \mathrm{SO}(\mathfrak{g}, \mathfrak{e})) d \operatorname{Vol}_{\mathfrak{g}},
$$

where $\mathrm{SO}(\mathfrak{g}, \mathfrak{e})_{p}$ is the set of orientation preserving isometries $T_{p} \mathcal{M} \rightarrow \mathbb{R}^{n}$, and the distance is measured with respect to the inner-product norm on $T_{p}^{*} \mathcal{M} \otimes \mathbb{R}^{n}$ induced by $\mathfrak{g}_{p}$ and the Euclidean metric e. Representing all of the above in a positive orthonormal basis at $T_{p} \mathcal{M}, \mathrm{SO}(\mathfrak{g}, \mathfrak{e})_{p}$ and dist reduces to $\mathrm{SO}(n)$ and the Frobenius distance. The notation $f_{\mathcal{M}}$

means the integral normalized by the volume, that is $f_{\mathcal{M}} f d \mathrm{Vol}_{\mathfrak{g}}:=\frac{1}{\operatorname{Vol}_{\mathfrak{g}}(\mathcal{M})} \int_{\mathcal{M}} f d \operatorname{Vol}_{\mathfrak{g}}$; this will be important as we consider the elastic energies of a family of shrinking manifolds.

The definition of $E_{\mathcal{M}}$ suggest a second notion of incompatibility - $(\mathcal{M}, \mathfrak{g})$ is incompatible if inf $E_{\mathcal{M}}>0$ even in the absence of boundary conditions. In [LP11, Theorem 2.2] it was shown that this is equivalent to the first (geometric) notion of incompatibility $\inf E_{\mathcal{M}}=0$ if and only if $\mathcal{R} \equiv 0$, where $\mathcal{R}$ is the Riemann curvature tensor of $\mathfrak{g}$ (see also [KMS] for a more general result between arbitrary manifolds).

Intuitively, one expect that the "more curvature" a body has, the less it is compatible with $\mathbb{R}^{n}$, and therefore the energy $E_{\mathcal{M}}$ would be higher. A natural question is therefore to make the previous result quantitative - to find a lower bound on the energy in terms of the curvature. This problem is highly non-trivial. First, it is a global problem as it involves the entire geometry of the manifold. second, $E_{\mathcal{M}}$ does not depend explicitly on the curvature, as the integrand involves only the metric $g$ and not its derivatives. The only general result we are aware of is [KS12], which gives a lower bound in terms of the scalar curvature for positively curved manifolds (and in dimension 2 for general manifolds). However, this bound is not very explicit, and in particular it is quite difficult to obtain from it effective bounds for thin elastic bodies, which are the main focus of this paper. These are described in the next section.

\subsection{Thin elastic bodies}

Much of the research in non-Euclidean elasticity, both in the physics and mathematics literature, is concerned with thin elastic bodies, i.e. bodies that have one or more slender 
dimensions. These include plate/shell theory and rod theory, corresponding to one and two slender dimensions (out of 3), respectively. The goal of these theories is to obtain the asymptotic behavior of the thin body as the thickness tends to zero.

Mathematically, the problem can be formulated as follows: Let $\left(\mathcal{M}^{n}, \mathfrak{g}\right)$ be a Riemannian manifold. For simplicity, assume that $g$ is smooth (though for the results in this paper $C^{2}$ would suffice). Let $\mathcal{S}^{k} \subset \mathcal{M}^{n}$ be a compact $k$-dimensional oriented submanifold with Lipschitz boundary. $\mathcal{S}$ is the mid-surface of the thin elastic body. The thin elastic body $\mathcal{S}_{h}$ is the $h$-tubular neighborhood of $\mathcal{S}$ in $\mathcal{M}$. More precisely, let $\left.T \mathcal{M}\right|_{\mathcal{S}}=T \mathcal{S} \oplus \mathcal{N} \mathcal{S}$ be the natural orthogonal decomposition, $\mathcal{N} S$ being the normal bundle of $\mathcal{S}$, and define

$$
\mathcal{S}_{h}:=\left\{\exp _{p}(v): p \in \mathcal{S}, v \in \mathcal{N} \mathcal{S},|v| \leq h\right\} .
$$

Two main (and interconnected) problems in the study of such bodies are finding the natural scaling of inf $E_{\delta_{h}}$ as $h \rightarrow 0$ (typically inf $E_{\delta_{h}} \sim h^{\beta}$ for some $\beta \geq 0$ ); and finding the limit of $\mathfrak{h}^{-\beta} E_{\delta_{h}}$ as $h \rightarrow 0$, which gives an effective elastic energy model for the mid-surface. In the mathematics community, the last question is typically treated in the framework of $\Gamma$-convergence (based on the seminal results in the Euclidean case [FJM02, FJM06]). We summarize below some of the main results in dimension reduction of non-Euclidean bodies that are relevant to this work (this does not aim to be a complete bibliography of the subject).

General dimension and codimension In [KS14] a general $\Gamma$-convergence result was proved for any dimension and co-dimension, for the scaling $\beta=2$. A corollary of their result is that inf $E_{\mathcal{S}_{h}}=O\left(h^{2}\right)$ if and only if there exists $F \in W^{2,2}\left(\mathcal{S} ; \mathbb{R}^{n}\right)$ and $q^{\perp} \in W^{1,2}\left(\mathcal{S} ; \mathcal{N S} \mathcal{S}^{*} \otimes \mathbb{R}^{n}\right)$ such that $d F \oplus q^{\perp} \in \mathrm{SO}(\mathfrak{g}, \mathfrak{e})$.

Plates/shells $(n=3, k=2)$ The case of plates and shells was initially treated in [LP11, BLS16], for the scaling $\beta=2$. Their results show that inf $E_{\mathcal{S}_{h}}=O\left(h^{2}\right)$ if and only if $\mathcal{S}$ can be $W^{2,2}$ isometrically immersed in $\mathbb{R}^{3}$ (this is a special case of the results of [KS14] mentioned above, in which the existence of $q^{\perp}$ follows from the existence of the isometric immersion F). In [BLS16, LRR] it was shown, under the assumption that the metric $g$ does not change along the thin dimension, that inf $E_{\mathcal{S}_{h}}=o\left(h^{2}\right)$ if and only if

$$
\left|\mathcal{R}_{1212}\right|=\left|\mathcal{R}_{1213}\right|=\left|\mathcal{R}_{1223}\right|=0,
$$

where $\mathcal{R}$ is the curvature tensor of $\mathcal{M}$ and the first two coordinates parametrize the mid surface. Furthermore, they proved that in this case inf $E_{S_{h}}=O\left(h^{4}\right)$, and that if $\inf E_{\mathcal{S}_{h}}=o\left(h^{4}\right)$ then the whole curvature tensor $\mathcal{R} \equiv 0$ on $\mathcal{S}$. The assumption that $\mathfrak{g}$ does not change along the thin dimension then implies that $\mathcal{R} \equiv 0$ everywhere, hence $\inf E_{S_{h}}=0$ in this case.

We also note that in [LRR] a complete $\Gamma$-convergence result for $h^{-4} E_{\mathcal{S}_{h}}$ is proved. See also [ALL17] for other recent results in the $O\left(h^{2}\right)$ regime, as well as numerous results in the physics literature for this scaling, e.g. [SRS07, ESK09b, ESK09a, ESK11]. Other scalings can be obtained due to external forces [BK14], or singular metrics [Olb17, COT17], but these are further away from the context of this paper.

Rods ( $n=3, k=1)$ For the rod case, it was shown in [KS14, Section 8.2] that $\inf E_{\delta_{h}}=o\left(h^{2}\right)$. It was later shown, by an uncontrolled formal expansion, that one expects inf $E_{\delta_{h}}=O\left(h^{4}\right)$ for a general non-Euclidean rod [AAE ${ }^{+} 12$ ]. 
Some recent results on non-Euclidean rods include [CRS17, KO18]; in both of them the setting is slightly different from ours, which results in a natural energy scaling of $h^{2}$ (rather than $h^{4}$ ). This is due to external forces in [CRS17] or rougher metrics in [KO18].

Other limits In [AKM ${ }^{+} 16$ the case of a body which is thin in all dimensions was considered, which corresponds to the case $k=0$, i.e. $\mathcal{S}=\{p\}$ (in this paper's framework); in other words, to the "local" elastic energy around a point. There they show, by an uncontrolled formal expansion, that inf $E_{\mathcal{S}_{h}} \sim h^{4}$, unless the Riemannian curvature at $p$ is zero.

When there are external forces or boundary conditions that imply that inf $E_{\mathcal{S}_{h}} \sim 1$, the dimensionally-reduced limit is called the membrane limit. In the context of incompatible elasticity, a $\Gamma$-convergence derivation of the membrane limit for every dimension and codimension was obtained in [KM14] (following the Euclidean case [LDR95, LDR96]); this is further away from the context of this paper because of the stretching boundary conditions.

\subsection{Main results}

In this paper we generalize the relations between curvature and energy scaling of thin plates [BLS16, LRR], to every dimension and co-dimension. Our results provide a unifying ground for most of the results mentioned above.

We start by proving a $\Gamma$-convergence result for the energies of shrinking balls around a point; we later "lift" this result to a general submanifold $\mathcal{S}$. Let $B_{h}(p)$ denote the ball of radius $h$ around a point $p \in \mathcal{M}$. We show the functionals $h^{-4} E_{B_{h}(p)} \Gamma$-converge to the functional

$$
I_{\mathcal{R}}: W^{1,2}\left(B, \mathbb{R}^{n}\right) \rightarrow \mathbb{R}, \quad I_{\mathcal{R}}[f]=\int_{B}\left|\operatorname{Sym} d f-\frac{1}{6} \mathcal{R}_{k i j l} x^{k} x^{l}\right|^{2}
$$

where $\mathcal{R}_{k i j l}$ are the components of the Riemann curvature tensor at $p$ for some choice of an orthonormal basis at $p, B$ is the unit ball in Euclidean space, and Sym $d f$ is the symmetric gradient $(\operatorname{Sym} d f)_{i j}=\partial_{i} f^{k} \delta_{k j}+\partial_{j} f^{k} \delta_{k i}$. Note that minimizing $I_{\mathcal{R}}$ is equivalent to a pure-traction linear elastic problem in the ball, with smooth body and traction forces (see [Cia88, Section 6.3]). The exact formulation of the $\Gamma$-convergence result is given in Theorem 2.1, after introducing some required notations.

Using this $\Gamma$-convergence result, we prove the following theorem:

Theorem 1.1

$$
\lim _{h \rightarrow 0} \frac{1}{h^{4}} \inf E_{B_{h}(p)}=\left|\mathcal{R}_{p}\right|^{2},
$$

where $|\cdot|$ is an inner-product induced norm on the subspace of $\left(T_{p}^{*} \mathcal{M}\right)^{3} \otimes T_{p} \mathcal{M}$ containing the possible curvature tensors at $p$. This norm is defined, in normal coordinates centered at $p$, as $|\mathcal{R}|:=\sqrt{\min I_{\mathcal{R}}}$, where $I_{\mathcal{R}}$ is defined in (1.3).

Remark: Note that $|\cdot|$, being an inner-product induced norm on a finite dimensional space, is of the form $\left|\mathcal{R}_{p}\right|^{2}=a^{i j k l a b c d} \mathcal{R}_{i j k l} \mathcal{R}_{a b c d}$, where $\mathcal{R}_{i j k l}$ are the components of $\mathcal{R}_{p}$ in some orthonormal basis in $T_{p} \mathcal{M}$. (1.3) implies that the constants $a^{i j k l a b c d}$ do not depend 
on $p$, and in this sense the norm is "point-independent". In particular, the map $p \mapsto\left|\mathcal{R}_{p}\right|$ is continuous.

Theorem 2.1 and Theorem 1.1 provide a "local" estimate of the infimal elastic energy in terms of the curvature. Moreover, they prove the correctness of the formal asymptotics derived in $\left.\mathrm{AKM}^{+} 16\right]$.

We then proceed to prove our main theorem regarding thin manifolds, thus establishing the relations between curvature and energy scaling of thin bodies in general dimension and co-dimension:

Theorem 1.2 1. [KS14]: There exists $F \in W^{2,2}\left(\mathcal{S} ; \mathbb{R}^{n}\right)$ and $q^{\perp} \in W^{1,2}\left(\mathcal{S} ; \mathcal{N} \mathcal{S}^{*} \otimes \mathbb{R}^{n}\right)$ such that $d F \oplus q^{\perp} \in S O(\mathfrak{g}, \mathfrak{e})$ a.e. if and only if

$$
\inf E_{S_{h}}=O\left(h^{2}\right)
$$

2.

$$
\inf E_{\delta_{h}}=o\left(h^{2}\right)
$$

if and only if there exist smooth maps $F: \mathcal{S} \rightarrow \mathbb{R}^{n}$ and $q^{\perp}: \mathcal{N S} \rightarrow \mathbb{R}^{n}$ such that $d F \oplus q^{\perp} \in S O(\mathfrak{g}, \mathrm{e})$ and $\nabla q^{\perp}=-d F \circ I I_{S, \mathcal{N}}$, where $I_{\mathcal{S}, \mathcal{M}}$ is the second fundamental form (the shape operator) of $\mathcal{S}$ in $\mathcal{M}$. In particular, using appropriate identifications (given by $F$ and $\left.q^{\perp}\right), I I_{F(\mathcal{S}), \mathbb{R}^{n}}$ coincides with $I I_{\mathcal{S}, \mathbb{M}}$ - in this sense, the first and second forms of $\mathcal{S}$ satisfy the Gauss-Codazzi-Ricci equations in $\mathbb{R}^{n}$. Moreover, (1.6) implies that

$$
\inf E_{\mathcal{S}_{h}}=O\left(h^{4}\right)
$$

3. (1.6) further implies that $\mathcal{R}^{\mathcal{M}}(X, Y)=0$ for every $X, Y \in T S 1$ If $\mathcal{S}$ is simply connected, then the converse also holds.

4.

$$
\inf E_{\mathcal{S}_{h}} \geq c h^{4} f_{S}\left|\mathcal{R}^{\mathcal{M}}\right|^{2} d V o l_{\left.\mathfrak{g}\right|_{\mathcal{S}}}+o\left(h^{4}\right)
$$

where $\left|\mathcal{R}^{\mathcal{M}}\right|$ is a norm on the curvature, defined below in Theorem 1.1 and $c$ is a universal constant. In particular, if

$$
\inf E_{\mathcal{S}_{h}}=o\left(h^{4}\right),
$$

then $\left.\mathcal{R}^{\mathcal{M}}\right|_{\delta} \equiv 0$, that is $\mathcal{R}^{\mathcal{M}}(X, Y)=0$ for every $X,\left.Y \in T \mathcal{N}\right|_{\delta}$. Furthermore, if (1.9) holds, $\mathcal{S}$ is simply-connected and $\mathcal{R}^{\mathcal{M}}$ is parallel along a foliation of curves emanating from $\mathcal{S}$, we have that for small enough $h, \mathcal{S}_{h}$ can be isometrically immersed in $\mathbb{R}^{n}$, hence inf $E_{\mathcal{S}_{h}}=0$.

We note that in the physically-interesting special case of rods $(k=1)$, Theorem 1.2 takes a particularly simple form:

Corollary 1.3 If $\operatorname{dim} \mathcal{S}=1$, then $\inf E_{\mathcal{S}_{h}}=O\left(h^{4}\right)$. If $\inf E_{\mathcal{S}_{h}}=o\left(h^{4}\right)$, then $\left.\mathcal{R}^{\mathcal{M}}\right|_{\mathcal{S}} \equiv 0$.

This proves the correctness of the scaling that appeared in [AAE ${ }^{+} 12$ ].

Part 1 of the Theorem 1.2 is merely a restatement of a corollary of the main result of [KS14], which we include for completeness. Parts 2 and 3 generalize the conditions for a scaling of $o\left(h^{2}\right)$ in [BLS16, LRR]; they clarify the geometric implications of this scaling also in the plate case. These are proved by carefully analyzing the limit functional

\footnotetext{
${ }^{1}$ Note that this does not imply that $\mathcal{S}$ is flat, which is $\mathcal{R}^{\mathcal{S}} \equiv 0$.
} 
obtained in [KS14]. We prove part 4 by using Theorem 1.1; more accurately, we need a slightly stronger version of it, Theorem 2.3 , which allows for perturbations of the centers of the balls.

We note that the choice of the energy (1.1) is for the sake of simplicity alone; all the results and proofs will hold (with some natural adjustments) for a more general energy density $W: T^{*} \mathcal{M} \otimes \mathbb{R}^{n} \rightarrow[0, \infty)$ as long as $W$ is $C^{2}$ near $\operatorname{SO}(\mathfrak{g}, \mathfrak{e})$ and

$$
\left.W\right|_{\mathrm{SO}(\mathrm{g}, \mathrm{e})}=0, \quad W(A) \geq c \operatorname{dist}^{2}(A, \mathrm{SO}(\mathfrak{g}, \mathrm{e})), \quad W(R A)=W(A),
$$

for some $c>0$, and every $R \in \mathrm{SO}(n)$.

Open questions We list below several questions that arise in the context of this work, which are however not in of the scope of this paper; they will be considered in future works.

1. The asymptotic analysis in $\left[\mathrm{AKM}^{+} 16\right]$ suggests that if one replaces $\mathbb{R}^{n}$ with a general ambient manifold, $h^{-4} \inf E_{B_{h}(p)}$ converges to a norm of the difference between the curvature at $p$ and the curvature at a point in the ambient manifold. It would be very interesting to generalize Theorem 1.1 to this case.

2. In the last part of Theorem 1.2 we proved that $\left.\mathcal{R}^{\mathcal{M}}\right|_{\mathcal{S}} \equiv 0$ is a necessary condition for the scaling $\inf E_{\mathcal{S}_{h}}=o\left(h^{4}\right)$. We suspect that for a sufficient condition, one might also require that $\nabla \mathcal{R}^{\mathcal{M}}(X, Y) \equiv 0$ for $X, Y \in T \mathcal{S}$. Obtaining a sufficient condition would require other tools than the ones used in this paper.

3. In this paper we only calculate the $\Gamma$-limit of $h^{-4} E_{\delta_{h}}$ for the case where $\mathcal{S}$ is a point; for plates, this was done in [LRR]. A natural question is to calculate this for any dimension and codimension, in the spirit of the limit of $h^{-2} E_{\delta_{h}}$ done in [KS14]. This would also give the exact limit of $h^{-4}$ inf $E_{\mathcal{S}_{h}}$ rather than the nonoptimal bound (1.8), and will also answer question 2 above. This general question seems, however, a pretty convoluted problem (even more than [KS14]); a more approachable yet interesting partial result would be to prove this $\Gamma$-limit for nonEuclidean rods.

Structure of this paper The paper is organized as follows: in Section 2 we consider the "local" problem of dimension reduction of small balls. We first state the $\Gamma$-convergence result (Theorem 2.1), and show that the scaling of $h^{4}$ is indeed the natural one (Section 2.1). We then prove Theorem 2.1 and Theorem 1.1. In Section 3 we prove Theorem 1.2 through a sequence of lemmas; those in Section 3.1 are more geometric and deal with the parts involving the $o\left(h^{2}\right)$ scaling; those in Section 3.2 are more analytic and deal with the $O\left(h^{4}\right)$ scaling.

Acknoledgments We thank Robert Jerrard for some useful advice and suggestions during the preparation of this paper, and Raz Kupferman for his critical reading of the manuscript. The second author was partially funded by the Israel Science Foundation (Grant No. 661/13), and by a grant from the Ministry of Science, Technology and Space, Israel and the Russian Foundation for Basic Research, the Russian Federation. 


\section{T-limit of the elastic energy of shrinking balls}

This section is concerned with the "local" problem - the $\Gamma$-convergence of elastic energies of small balls around a point (Theorem 2.1) and the limit of their infima (Theorem 1.1). As mentioned in the introduction, we shall prove a slightly stronger version of Theorem 1.1 which allows for perturbations (Theorem 2.3 below): Instead of considering the behavior of $E_{B_{h}(p)}$, we shall consider the behavior of $E_{B_{h}\left(p_{h}\right)}$ where $p_{h}$ is a sequence in $\mathcal{M}$ converging to $p$. We begin by introducing some notations.

- Fix $h_{0}<\operatorname{inj}(p)$, so that $\exp _{p}: B_{h_{0}}(0) \subset T_{p} \mathcal{M} \rightarrow B_{h_{0}}(p)$ is a diffeomorphism, where $B_{h_{0}}(0)$ is the ball of radius $h_{0}$ centered at the origin in $T_{p} \mathcal{M}$, and $B_{h_{0}}(p)$ is the ball of radius $h_{0}$ around $p$ in $\mathcal{M}$. For some small enough neighborhood $U$ of $p$, $\exp _{q}: B_{h_{0}}(0) \subset T_{q} \mathcal{M} \rightarrow B_{h_{0}}(q)$ is also a diffeomorphism for every $q \in U$, and the $\operatorname{map}(q, v) \mapsto \exp _{q}(v)$ is smooth.

- Fix a smooth orthonormal frame $F$ of $\left.T \mathcal{M}\right|_{U}$. For every $q \in U$, we identify $T_{q} \mathcal{M} \sim \mathbb{R}^{n}$ using $F_{q}$; in particular, this identifies $B_{h}(0) \subset T_{q} \mathcal{M}$ with $B_{h}(0) \subset \mathbb{R}^{n}$. Using this identification, $\exp _{q}$ defines normal coordinates on $B_{h_{0}}(q)$. Note that the components $\mathcal{R}_{i j k l}(q)$ of the Riemann curvature tensor in this coordinate system (centered at $q$ ) are the components of the curvature tensor with respect to $F$ at $q$. In particular, the map $q \mapsto \mathcal{R}_{i j k l}(q)$ is smooth.

- For $q \in U$, denote $\iota_{q}:=\exp _{q}^{-1}: B_{h_{0}}(q) \rightarrow T_{q} \mathcal{M} \sim \mathbb{R}^{n}$; this is the identity map in the above normal coordinates (centered at $q$ ). With a slight abuse of notation we will consider $\iota_{q}$ also with a restricted domain $B_{h}(q)$ for some $h<h_{0}$.

- For a map $u: B_{h}(q) \rightarrow \mathbb{R}^{n}$, define the rescaled map $\tilde{u}: B \rightarrow \mathbb{R}^{n}$ by $\tilde{u}(x):=u(h x)$, where $B:=B_{1}(0) \subset \mathbb{R}^{n}$, using normal coordinates. Note that we view $\tilde{u}$ as a map between Euclidean spaces.

- Unless otherwise noted, all integral norms (e.g. $\left.L^{2}, W^{1,2}\right)$ are normalized by the volume of the relevant domain.

Theorem 2.1 Let $p_{h} \in \mathcal{M}$ be a sequence converging to $p$. Then the following hold:

1. Compactness and lower semicontinuity: Assume that $u_{h} \in W^{1,2}\left(B_{h}\left(p_{h}\right) ; \mathbb{R}^{n}\right)$ satisfy $E_{B_{h}\left(p_{h}\right)}\left[u_{h}\right]=O\left(h^{4}\right)$. Then

(a) Rigidity: There exists $Q_{h} \in S O(n)$ and $c_{h} \in \mathbb{R}^{n}$ such that the maps $\bar{u}_{h}=Q_{h} u_{h}-c_{h}$ satisfy $\left\|\bar{u}_{h}-\iota_{p_{h}}\right\|_{W^{1,2}\left(B_{h}\left(p_{h}\right) ; \mathbb{R}^{n}\right)}=O\left(h^{2}\right)$.

(b) Compactness: The "displacements" $v_{h}=\bar{u}_{h}-\iota_{p_{h}}$ converge (modulo a subsequence), after rescaling, to some $f \in W^{1,2}\left(B, \mathbb{R}^{n}\right)$, in the following sense 2

$$
\frac{1}{h^{3}} d \tilde{v}_{h} \rightarrow d f \text { weakly in } L^{2} .
$$

(c) Lower semicontinuity: if $v_{h} \rightarrow f$ in the above sense, then

$$
\liminf \frac{1}{h^{4}} E_{B_{h}\left(p_{h}\right)}\left[u_{h}\right] \geq I_{\mathcal{R}}(f) .
$$

\footnotetext{
${ }^{2}$ Note that for different choices of $Q_{h}$ we can have that $v_{h}$ converge to different functions; however we can further require that $\int_{B} f=0, \int_{B} \operatorname{Skew}(d f)=0$. In this case there is no ambiguity.
} 
2. Recovery sequence: for every $f \in W^{1,2}\left(B, \mathbb{R}^{n}\right)$, there exists a sequence $u_{h} \in W^{1,2}\left(B_{h}\left(p_{h}\right) ; \mathbb{R}^{n}\right)$ such that $v_{h}=u_{h}-\iota_{p_{h}}$ converges strongly to $f$ (in the sense of (2.1)), and

$$
\frac{1}{h^{4}} E_{B_{h}\left(p_{h}\right)}\left[u_{h}\right] \rightarrow I_{\mathcal{R}}(f)
$$

\subsection{The energy scaling of the exponential map}

In this section we prove an upper bound of inf $E_{B_{h}\left(p_{h}\right)}$, by using the exponential map. This yield the optimal scaling with $h$, though not the optimal constant.

Lemma 2.2 (The asymptotic distortion of the exponential map) For every $q \in U$, the inverse exponential map $\iota_{q}$ satisfies $E_{B_{h}(q)}\left[\iota_{q}\right]<C h^{4}$ for some $C>0$ independent of $q$. In particular, for the sequence $p_{h} \rightarrow p$ in Theorem 2.1] inf $E_{B_{h}\left(p_{h}\right)}=O\left(h^{4}\right)$.

Proof: The energy density $\operatorname{dist}(d u, \mathrm{SO}(\mathfrak{g}, \mathrm{e}))$ satisfies

$$
\operatorname{dist}(d u, \mathrm{SO}(\mathfrak{g}, \mathfrak{e}))=\operatorname{dist}\left(d u \circ A^{-1}, \mathrm{SO}(n)\right)
$$

for every $A \in \mathrm{SO}(\mathfrak{g}, \mathfrak{e})$, where in the right-hand side, the distance is with respect to the Frobenius norm on $\mathbb{R}^{n} \otimes \mathbb{R}^{n}$. In particular, for an orientation preserving map $u$ we have

$$
\operatorname{dist}(d u, \mathrm{SO}(\mathfrak{g}, \mathrm{e}))=\left|\sqrt{\left(d u A^{-1}\right)^{T} d u A^{-1}}-\mathrm{Id}\right|,
$$

where the transpose on the right-hand side is the "standard" (Euclidean) transpose (since $d u \circ A^{-1}: \mathbb{R}^{n} \rightarrow \mathbb{R}^{n}$ ). We denote by $\mathfrak{g}_{q}(x)$ the matrix representation of the metric $\mathfrak{g}$ at a point $x$ with respect to the normal coordinates centered at $q$, and denote by $\sqrt{\mathfrak{g}_{q}}(x)$ the positive square root of this matrix. It is well known that $\sqrt{\mathfrak{g}_{q}} \in \mathrm{SO}(\mathfrak{g}, \mathrm{e})$, where both sides are evaluated at $x$. Applying (2.3) with $A=\sqrt{\mathfrak{g}_{q}}$ and $u=\iota_{q}$, and using the fact that $\iota_{q}$ is the identity map in normal coordinates, we have that

$$
\operatorname{dist}\left(d \iota_{q}, \mathrm{SO}(\mathfrak{g}, \mathfrak{e})\right)=\left|{\sqrt{\mathfrak{g}_{q}}}^{-1}-\mathrm{Id}\right|
$$

In normal coordinates, we further have

$$
\left(\mathfrak{g}_{q}\right)_{i j}(x)=\delta_{i j}+\frac{1}{3} \mathcal{R}_{k i j l}(q) x^{k} x^{l}+O\left(|x|^{3}\right),
$$

and therefore

$$
\left({\sqrt{\mathfrak{g}_{q}}}^{-1}\right)^{i j}(x)=\delta^{i j}-\frac{1}{6} \mathcal{R}_{k i j l}(q) x^{k} x^{l}+O\left(|x|^{3}\right) .
$$

where $\mathcal{R}_{k i j l}(q)$ are the components of the Riemannian curvature tensor at $q$. Note that our choice of coordinates implies that the remainders $O\left(|x|^{3}\right)$ (and similar remainders below) can bounded independently of $q \in U$, that is $O\left(|x|^{3}\right)<C|x|^{3}$ for some $C>0$ independent of $q$. Therefore we obtain

$$
\operatorname{dist}^{2}\left(d_{l_{q}}, \mathrm{SO}\left(\mathfrak{g}_{q}, \mathfrak{e}\right)\right)=\left|\left({\sqrt{\mathfrak{g}_{q}}}^{-1}\right)^{i j}-\delta^{i j}\right|^{2}=\frac{1}{36} \delta^{i a} \delta^{j b} \mathcal{R}_{k i j l}(q) \mathcal{R}_{c a b d}(q) x^{k} x^{l} x^{c} x^{d}+O\left(|x|^{5}\right)
$$

The volume form in coordinates reads

$$
d \operatorname{Vol}_{\mathfrak{g}}=\sqrt{\operatorname{det}\left(\mathfrak{g}_{q}\right)} d x=\left(1+O\left(|x|^{2}\right)\right) d x
$$


Plugging those expressions into the functional, and noting that the domain $B_{h}(q)$ is in normal coordinates the Euclidean ball $B_{h}(0)$, we obtain that

$$
\begin{aligned}
E_{B_{h}(q)}\left[\iota_{q}\right] & =\int_{B_{h}(0)}\left(\frac{1}{36} \delta^{i a} \delta^{j b} \mathcal{R}_{k i j l}(q) \mathcal{R}_{c a b d}(q) x^{k} x^{l} x^{c} x^{d}+O\left(|x|^{5}\right)\right) d x \\
& =\delta^{i a} \delta^{j b} \mathcal{K}^{k l c d} \mathcal{R}_{k i j l}(q) \mathcal{R}_{c a b d}(q) h^{4}+O\left(h^{5}\right),
\end{aligned}
$$

where

$$
\kappa^{k l c d}:=\frac{1}{36} f_{B_{1}(0)} x^{k} x^{l} x^{c} x^{d} d x
$$

This estimate completes the proof, since $\mathcal{R}_{k i j l}(q)$ can be bounded uniformly in $q$.

Remark: The map $\iota_{q}$ is not optimal - a direct calculation shows that by perturbing it one can get a lower $h^{4}$-coefficient than in (2.7). Specifically, this can be done using $u_{h}(x)=x+P(x)$, where $P$ is a vector of homogeneous polynomials of degree 3 .

\subsection{Proof of Theorem 2.1 ( $\Gamma$-convergence)}

In this section we prove Theorem 2.1. Throughout the proof, we will consider maps $A \in T_{q}^{*} \mathcal{M} \otimes \mathbb{R}^{n}$ for some $q \in B_{h}\left(p_{h}\right)$ (for example, $d u_{h}(q)$ for $u_{h} \in W^{1,2}\left(B_{h}\left(p_{h}\right) ; \mathbb{R}^{n}\right)$ ). As discussed before, $T_{q}^{*} \mathcal{M} \otimes \mathbb{R}^{n}$ has a natural inner-product induced by the metrics $g$ and $e$, with respect to we can consider $|A|, \operatorname{dist}(A, \mathrm{SO}(\mathfrak{g}, \mathfrak{e}))$, etc.

However, using the normal coordinates considered before, it would be useful to view $A$ also as a map $\mathbb{R}^{n} \rightarrow \mathbb{R}^{n}$, where both the domain and target are endowed with the Euclidean metric e. Henceforth, whenever we say that we consider $A$ as a map $\mathbb{R}^{n} \rightarrow \mathbb{R}^{n}$, the norm we take is the Euclidean norm, and similarly we consider its distance (in $\mathbb{R}^{n} \otimes \mathbb{R}^{n}$ ) from $\mathrm{SO}(n)$.

By (2.4), it follows that for every $A \in T_{q}^{*} \mathcal{M} \otimes \mathbb{R}^{n}$ and $q \in B_{h}\left(p_{h}\right)$ the metrics are equivalent with a uniform constant, that is

$$
\frac{|A|_{T_{q}^{*} \mathcal{M} \otimes \mathbb{R}^{n}}}{|A|_{\mathbb{R}^{n} \otimes \mathbb{R}^{n}}}=1+O\left(h^{2}\right) .
$$

Therefore, in most cases it would not matter if we use $|A|_{T_{q}^{*} \mathcal{M} \otimes \mathbb{R}^{n}}$ or $|A|_{\mathbb{R}^{n} \otimes \mathbb{R}^{n}}$. In these cases, we simply write $|A|$. To simplify notation, we will also write $E_{h}$ instead of $E_{B_{h}\left(p_{h}\right)}$.

\subsubsection{Rigidity (part 1a)}

The proof of this part is a direct application of the Friesecke-James-Müller rigidity theorem [FJM02, Theorem 3.1], taking into account that our metric is not Euclidean, but not far from it on small balls.

Let $u_{h} \in W^{1,2}\left(B_{h}\left(p_{h}\right) ; \mathbb{R}^{n}\right)$. In normal coordinates centered at $p_{h}$, we can consider $u_{h}$ as a map $B_{h}(0) \rightarrow \mathbb{R}^{n}$ between Euclidean spaces. By the Friesecke-James-Müller rigidity theorem [FJM02, Theorem 3.1], there exist a constant $C>0$ (independent of $u_{h}$ and $h$ ), and matrices $Q_{h} \in \mathrm{SO}(n)$ such that

$$
f_{B_{h}(0)}\left|Q_{h} d u_{h}-\mathrm{Id}\right|^{2} d x \leq C f_{B_{h}(0)} \operatorname{dist}^{2}\left(d u_{h}, \mathrm{SO}(n)\right) d x
$$


Where distances and volume form are with respect to the Euclidean metric (not with respect to $\mathfrak{g}$ ), as discussed above. By (2.6), we have that integrating with respect to $d x$ or $d \mathrm{Vol}_{\mathfrak{g}}$ is the same up to a multiplicative constant independent of $h$. By (2.8), the $T_{q}^{*} \mathcal{M} \otimes \mathbb{R}^{n}$ and $\mathbb{R}^{n} \otimes \mathbb{R}^{n}$ norms on $Q_{h} d u_{h}$ - Id are equivalent, with a constant independent of $h$. Using these, and the fact that $\iota_{p_{h}}$ is the identity map in coordinates, we can write the above inequality as

$$
f_{B_{h}\left(p_{h}\right)}\left|d\left(Q_{h} u_{h}\right)-d l_{p_{h}}\right|^{2} d \operatorname{Vol}_{\mathfrak{g}} \leq C f_{B_{h}(0)} \operatorname{dist}^{2}\left(d u_{h}(x), \mathrm{SO}(n)\right) d \operatorname{Vol}_{\mathfrak{g}}(x) .
$$

Note that the right-hand side is similar to $E_{h}\left[u_{h}\right]$, but not the same $-\operatorname{dist}^{2}\left(d u_{h}(x), \operatorname{SO}(n)\right)$ is the distance squared of the coordinate representation of $d u_{h}$ to $S O(n)\left(\right.$ in $\left.\mathbb{R}^{n} \times \mathbb{R}^{n}\right)$, while the integrand of $E_{h}\left[u_{h}\right]$ is the distance of $d u_{h}$ to $S O(g, e)$ in $T^{*} \mathcal{M} \otimes \mathbb{R}^{n}$. In order to complete the proof, we need to show the right-hand side is bounded by $C\left(E_{h}\left[u_{h}\right]+h^{4}\right)$, where $C>0$ is independent of $h, u_{h}$.

This follows from the following pointwise calculation. Let $q \in B_{h}\left(p_{h}\right)$ and let $T \in$ $T_{q}^{*} \mathcal{M} \otimes \mathbb{R}^{n}$. Let $\hat{T} \in \mathbb{R}^{n} \otimes \mathbb{R}^{n}$ be the matrix representation of $A$ in normal coordinates. We claim

$$
|\operatorname{dist}(T, \mathrm{SO}(\mathfrak{g}, \mathfrak{e}))-\operatorname{dist}(\hat{T}, \mathrm{SO}(n))| \leq C|T| h^{2}
$$

where each distance is considered with respect to its natural inner-product. The constant $C>0$ is independent of $q$ and $h$. Indeed, using (2.2) and the fact that $S \rightarrow \operatorname{dist}(S, \operatorname{SO}(n))$ is 1-Lipschitz (for maps $\mathbb{R}^{n} \rightarrow \mathbb{R}^{n}$ ), we have

$$
\begin{aligned}
|\operatorname{dist}(T, \mathrm{SO}(\mathfrak{g}, \mathfrak{e}))-\operatorname{dist}(\hat{T}, \mathrm{SO}(n))| & =\left|\operatorname{dist}\left(T \circ \sqrt{\mathfrak{g}}^{-1}, \mathrm{SO}(n)\right)-\operatorname{dist}(\hat{T}, \mathrm{SO}(n))\right| \\
& \leq|\hat{T}|\left|\sqrt{\mathrm{g}}^{-1}-\mathrm{Id}\right| \\
& \leq C|T| h^{2}
\end{aligned}
$$

where in the last line we used (2.5) and (2.8), centered at the point $p_{h}$. We therefore have

$$
\begin{aligned}
f_{B_{h}\left(p_{h}\right)} \operatorname{dist}^{2}\left(d u_{h}, \mathrm{SO}(n)\right) d \operatorname{Vol}_{\mathfrak{g}} & \leq f_{B_{h}\left(p_{h}\right)}\left(\operatorname{dist}\left(d u_{h}, \mathrm{SO}(\mathfrak{g}, \mathfrak{e})\right)+C\left|d u_{h}\right| h^{2}\right)^{2} d \operatorname{Vol}_{\mathfrak{g}} \\
& \leq f_{B_{h}\left(p_{h}\right)} C^{\prime}\left(\operatorname{dist}\left(d u_{h}, \mathrm{SO}(\mathfrak{g}, \mathfrak{e})\right)+h^{2}\right)^{2} d \operatorname{Vol}_{\mathfrak{g}} \\
& \leq 2 C^{\prime}\left(E_{h}\left[u_{h}\right]+h^{4}\right) .
\end{aligned}
$$

Together with (2.9), this shows that

$$
f_{B_{h}\left(p_{h}\right)}\left|d\left(Q_{h} u_{h}\right)-d \iota\right|^{2} \leq C\left(E_{h}\left[u_{h}\right]+h^{4}\right),
$$

for some constant $C>0$. Part 1a of Theorem 2.1 now follows by Poincaré inequality.

\subsubsection{Compactness and lower bound (parts $1 \mathrm{~b}$ and 1c)}

Suppose $E_{h}\left[u_{h}\right]=O\left(h^{4}\right)$ and let $\bar{u}_{h}=Q_{h} u_{h}-c_{h}$ as in part 1a of Thereom 2.1, such that $\left\|v_{h}\right\|_{W^{1,2}\left(B_{h}\left(p_{h}\right) ; \mathbb{R}^{n}\right)}=O\left(h^{2}\right)$, where $v_{h}=\bar{u}_{h}-\iota$. Let $\tilde{v}_{h} \in W^{1,2}\left(B ; \mathbb{R}^{n}\right)$ be the rescaling of $v_{h}$, that is $\tilde{v}_{h}(x):=v_{h}(h x)$. Note that $\left\|d \tilde{v}_{h}\right\|_{L^{2}}=O\left(h^{3}\right)$ (recall that the norms are normalized by 
the volume of the domain, and that the Euclidean and Riemannian norms are uniformly equivalent by (2.8) ). Therefore we have that

$$
\frac{1}{h^{3}} d \tilde{v}_{h} \rightarrow V \quad \text { in } L^{2}\left(B ; T^{*} B \otimes \mathbb{R}^{n}\right) .
$$

Note that $d\left(d \tilde{v}_{h}\right)=0$ in $W^{-1,2}\left(B ; \Lambda^{2} T^{*} B \otimes \mathbb{R}^{n}\right)$, by the weak Poincaré Lemma [Cia13, Theorem 6.17-4], hence also $d\left(h^{-3} d \tilde{v}_{h}\right)=0$. Since the weak convergence in $L^{2}\left(B ; T^{*} B \otimes \mathbb{R}^{n}\right)$ respects the weak formulation of the $d$ operator, we obtain that $d V=0$ in $W^{-1,2}$. Invoking the Poincaré Lemma again, we obtain that $V=d f$ for some $f \in W^{1,2}\left(B ; \mathbb{R}^{n}\right)$. This completes the proof of part $1 \mathrm{~b}$ (compactness).

We now prove part 1c, the lower bound for the energy. First, we write the energy density as

$$
\operatorname{dist}\left(d u_{h}, \mathrm{SO}(\mathfrak{g}, \mathfrak{e})\right)=\operatorname{dist}\left(d u_{h} \sqrt{\mathfrak{g}_{p_{h}}}-1, \mathrm{SO}(n)\right)=\operatorname{dist}\left(\operatorname{Id}+h^{2} G_{h}, \mathrm{SO}(n)\right),
$$

where

$$
G_{h} \in L^{2}\left(B_{h}\left(p_{h}\right) ; \mathbb{R}^{n} \otimes \mathbb{R}^{n}\right), \quad G_{h}:=\frac{d u_{h} \circ \sqrt{\mathfrak{g}_{p_{h}}}-1-\mathrm{Id}}{h^{2}} .
$$

Now, in coordinates we have (using $d u_{h}=\mathrm{Id}+d v_{h}$ )

$$
\frac{d u_{h} \circ \sqrt{\mathfrak{g}_{p_{h}}}-1-\mathrm{Id}}{h^{2}}=\frac{\left(\sqrt{\mathfrak{g}_{p_{h}}}-1-\mathrm{Id}\right)}{h^{2}}+\frac{d v_{h}}{h^{2}}+\frac{d v_{h}}{h^{2}}\left(\sqrt{\mathfrak{g}_{p_{h}}}-1-\mathrm{Id}\right)
$$

Since $\left\|{\sqrt{\mathfrak{g}_{p_{h}}}}^{-1}-\mathrm{Id}\right\|_{\infty}=O\left(h^{2}\right)$ and $\left\|v_{h}\right\|_{W^{1,2}\left(B_{h}\left(p_{h}\right) ; \mathbb{R}^{n}\right)}=O\left(h^{2}\right)$, we have $\left\|G_{h}\right\|_{2}=O(1)$. Let $\tilde{G}_{h} \in L^{2}\left(B ; \mathbb{R}^{n} \otimes \mathbb{R}^{n}\right)$ be the rescaling of $G_{h}$, that is $\tilde{G}_{h}(x)=G_{h}(h x)$. Since $\left\|G_{h}\right\|_{2}=O(1)$ we also have $\left\|\tilde{G}_{h}\right\|_{2}=O(1)$, hence $\tilde{G}_{h}$ weakly convergens in $L^{2}\left(B ; \mathbb{R}^{n} \otimes \mathbb{R}^{n}\right)$ to some $G$. From (2.12), (2.5) and (2.1) a direct calculation shows that

$$
G(x)=d f(x)-\frac{1}{6} \mathcal{R}_{k i j l}(p) x^{k} x^{l},
$$

using the continuity of $\mathcal{R}_{k i j l}\left(p_{h}\right) \rightarrow \mathcal{R}_{k i j l}(p)$.

Now, by Taylor expanding $\operatorname{dist}(\operatorname{Id}+A, \mathrm{SO}(n))$, it follows from (2.11) that

$$
\left.\left|\operatorname{dist}^{2}\left(d u_{h}, \mathrm{SO}(\mathfrak{g}, \mathrm{e})\right)-h^{4}\right| \frac{G_{h}+G_{h}^{T}}{2}\right|^{2} \mid \leq \omega\left(h^{2}\left|G_{h}\right|\right) .
$$

where $\omega(t)$ is a non-negative function satisfying $\lim _{t \rightarrow 0} \omega(t) / t^{2}=0$. Therefore we have

$$
\begin{aligned}
\frac{1}{h^{4}} E_{h}\left(u_{h}\right) & \geq f_{B_{h}\left(p_{h}\right)}\left(\left|\frac{G_{h}+G_{h}^{T}}{2}\right|^{2}-\frac{\omega\left(h^{2}\left|G_{h}\right|\right)}{h^{4}}\right) d \operatorname{Vol}_{\mathfrak{g}} \\
& \geq f_{B_{h}\left(p_{h}\right)} \chi_{h}\left(\left|\frac{G_{h}+G_{h}^{T}}{2}\right|^{2}-\frac{\omega\left(h^{2}\left|G_{h}\right|\right)}{h^{4}}\right) d \operatorname{Vol}_{\mathfrak{g}} \\
& =f_{B_{h}\left(p_{h}\right)}\left(\chi_{h}\left|\frac{G_{h}+G_{h}^{T}}{2}\right|^{2}-\chi_{h}\left|G_{h}\right|^{2} \frac{\omega\left(h^{2}\left|G_{h}\right|\right)}{h^{4}\left|G_{h}\right|^{2}}\right) d \operatorname{Vol}_{\mathfrak{g}}
\end{aligned}
$$

where

$$
\chi_{h}(x)= \begin{cases}1 & \left|G_{h}(x)\right|<h^{-1} \\ 0 & \left|G_{h}(x)\right| \geq h^{-1}\end{cases}
$$


Now, on the support of $\chi_{h}$ we have $h^{2}\left|G_{h}\right|<h$, and therefore, since $\left\|G_{h}\right\|_{2}=O(1)$, we have

$f_{B_{h}\left(p_{h}\right)} \chi_{h}\left|G_{h}\right|^{2} \frac{\omega\left(h^{2}\left|G_{h}\right|\right)}{h^{4}\left|G_{h}\right|^{2}} d \operatorname{Vol}_{\mathfrak{g}}=\frac{1}{\operatorname{Vol}\left(B_{h}\left(p_{h}\right)\right)} \int_{G_{h}<h^{-1}}\left|G_{h}\right|^{2} \frac{\omega\left(h^{2}\left|G_{h}\right|\right)}{h^{4}\left|G_{h}\right|^{2}} \leq\left\|G_{h}\right\|_{2} \sup _{t \in(0, h)} \frac{\omega(t)}{t^{2}} \rightarrow 0$.

Therefore,

$$
\begin{aligned}
\liminf \frac{1}{h^{4}} E_{h}\left(u_{h}\right) & \geq \liminf f_{B_{h}\left(p_{h}\right)} \chi_{h}\left|\frac{G_{h}+G_{h}^{T}}{2}\right|^{2} d \operatorname{Vol}_{\mathfrak{g}} \\
& =\liminf f_{B_{h}\left(p_{h}\right)}\left|\frac{\chi_{h} G_{h}+\chi_{h} G_{h}^{T}}{2}\right|^{2} d \operatorname{Vol}_{\mathfrak{g}} \\
& \stackrel{\text { 2.6) }}{=} \liminf f_{B_{h}(0)}\left|\frac{\chi_{h} G_{h}+\chi_{h} G_{h}^{T}}{2}\right|^{2} d x \\
& =\liminf f_{B}\left|\frac{\tilde{\chi}_{h} \tilde{G}_{h}+\tilde{\chi}_{h} \tilde{G}_{h}^{T}}{2}\right|^{2} d x .
\end{aligned}
$$

Since $\left\|G_{h}\right\|_{2}=O(1)$, we have that $\tilde{\chi}_{h} \rightarrow 1$ in $L^{2}$ (and uniformly bounded), and therefore $\tilde{G}_{h} \rightarrow G$ implies that $\tilde{\chi}_{h} \tilde{G}_{h} \rightarrow G$.

By passing to subsequences, we can always assume that $\tilde{G}_{h} \rightarrow G$ for a subsequence that achieves $\lim \inf \frac{1}{h^{4}} E_{h}\left(u_{h}\right)$. Therefore, by the lower semicontinuity of the norm under weak convergence, (2.15) implies

$$
\liminf \frac{1}{h^{4}} E_{h}\left(u_{h}\right) \geq f_{B}\left|\frac{G+G^{T}}{2}\right|^{2} d x \stackrel{\frac{2.13}{=}}{=} I_{\mathcal{R}}(f) .
$$

\subsubsection{Upper bound (part 2)}

We now prove part 2 of Theorem 2.1 - for every $f \in W^{1,2}\left(B, \mathbb{R}^{n}\right)$, there exists a sequence $u_{h} \in W^{1,2}\left(B_{h}\left(p_{h}\right) ; \mathbb{R}^{n}\right)$ such that $v_{h}=u_{h}-\iota_{p_{h}}$ converges strongly to $f$ (in the sense of (2.1)), and $h^{-4} E_{h}\left[u_{h}\right] \rightarrow I_{\mathcal{R}}[f]$.

Indeed, fix $f \in W^{1,2}\left(B, \mathbb{R}^{n}\right)$, and choose $f_{h} \in W^{1,2}\left(B ; \mathbb{R}^{n}\right)$ such that $f_{h} \rightarrow f$ and $\left\|d f_{h}\right\|_{\infty}<$ $h^{-1}$. Define, in coordinates centered at $p_{h}, u_{h}(x)=x+h^{3} f_{h}(x / h)$. Then obviously $v_{h}=u_{h}-\iota_{p_{h}}=h^{3} f_{h}(x / h)$ converges to $f$, and

$$
G_{h}(x):=\frac{d u_{h} \circ \sqrt{\mathfrak{g}_{p_{h}}}-1-\mathrm{Id}}{h^{2}}=\frac{1}{h^{2}}\left(h^{2} d f_{h}(x / h)-\frac{1}{6} \mathcal{R}_{k i j l}\left(p_{h}\right) x^{k} x^{l}\right)+O(h) .
$$

Therefore

$$
\tilde{G}_{h} \rightarrow d f(x)-\frac{1}{6} \mathcal{R}_{k i j l}(p) x^{k} x^{l} \quad \text { strongly in } L^{2} .
$$

Now, since $\left\|G_{h}\right\|_{\infty}=O\left(h^{-1}\right)$, we have from (2.11) and (2.14) that

$$
\left.\left.\left|\operatorname{dist}^{2}\left(d u_{h}, \mathrm{SO}(\mathfrak{g}, \mathfrak{e})\right)-h^{4}\right| \frac{G_{h}+G_{h}^{T}}{2}\right|^{2}\left|\leq \omega\left(h^{2}\left|G_{h}\right|\right)=h^{4}\right| G_{h}\right|^{2} \frac{\omega\left(h^{2}\left|G_{h}\right|\right)}{h^{4}\left|G_{h}\right|^{2}} \leq\left|G_{h}\right|^{2} o\left(h^{4}\right),
$$

hence

$$
\left.\left.\left|\frac{1}{h^{4}} E_{h}\left(u_{h}\right)-f_{B_{h}\left(p_{h}\right)}\right| \frac{G_{h}+G_{h}^{T}}{2}\right|^{2}\left|\leq o(1) f_{B_{h}\left(p_{h}\right)}\right| G_{h}\right|^{2}=o(1),
$$

and by (2.16) we obtain that $h^{-4} E_{h}\left[u_{h}\right] \rightarrow I_{\mathcal{R}}[f]$. 


\subsection{Proof of Theorem 1.1 (limit of infima)}

We shall now prove the slightly stronger version of Theorem 1.1, namely:

Theorem 2.3 Let $p_{h} \in \mathcal{M}$ be a sequence converging to $p$. Then

$$
\lim _{h \rightarrow 0} \frac{1}{h^{4}} \inf E_{B_{h}\left(p_{h}\right)}=\left|\mathcal{R}_{p}\right|^{2},
$$

Where $|\mathcal{R}|:=\sqrt{\min I_{\mathcal{R}}}$ is defined in normal coordinates centered at $p$.

So far we have shown that $h^{-4} E_{h} \Gamma$-converges to $I_{\mathcal{R}}$, including a compactness argument. In particular, a standard argument shows convergence of minimizers:

Lemma 2.4 Let $u_{h}$ be a sequence of approximate minimizers of $\frac{1}{h^{4}} E_{B_{h}\left(p_{h}\right)}$, that is

$$
\frac{1}{h^{4}} E_{B_{h}\left(p_{h}\right)}\left[u_{h}\right]=\inf _{W^{1,2}\left(B_{h}\left(p_{h}\right) ; \mathbb{R}^{n}\right)} \frac{1}{h^{4}} E_{B_{h}\left(p_{h}\right)}+o(1) .
$$

Then the associated displacements $v_{h}$ defined in Theorem 2.1 converge (modulo a subsequence) to a minimizer of $I_{R}$. In particular,

$$
\lim _{h \rightarrow 0} \inf _{W^{1,2}\left(B_{h}\left(p_{h}\right) ; \mathbb{R}^{n}\right)} \frac{1}{h^{4}} E_{B_{h}\left(p_{h}\right)}=\min _{W^{1,2}\left(B ; \mathbb{R}^{n}\right)} I_{\mathcal{R}} .
$$

Proof: By Lemma 2.2, inf $\frac{1}{h^{4}} E_{B_{h}\left(p_{h}\right)}=O(1)$, hence $E_{B_{h}\left(p_{h}\right)}\left[u_{h}\right]=O\left(h^{4}\right)$. Therefore, by Theorem 2.1, parts $1(\mathrm{~b})$ and $1(\mathrm{c}), v_{h}$ converges to $f \in W^{1,2}\left(B ; \mathbb{R}^{n}\right)$. Choose an arbitrary $f^{\prime} \in W^{1,2}\left(B ; \mathbb{R}^{n}\right)$, and let $u_{h}^{\prime}$ be a recovery sequence for $f^{\prime}$ according to part 2 of Theorem 2.1. We therefore have

$$
I_{\mathcal{R}}[f] \leq \lim _{h \rightarrow 0} \frac{1}{h^{4}} E_{B_{h}\left(p_{h}\right)}\left[u_{h}\right]=\lim _{h \rightarrow 0} \inf _{W^{1,2}\left(B_{h}\left(p_{h}\right) ; \mathbb{R}^{n}\right)} \frac{1}{h^{4}} E_{B_{h}\left(p_{h}\right)} \leq \lim _{h \rightarrow 0} \frac{1}{h^{4}} E_{B_{h}\left(p_{h}\right)}\left[u_{h}^{\prime}\right]=I_{\mathcal{R}}\left[f^{\prime}\right],
$$

hence $f$ is a minimizer. By choosing $f^{\prime}=f$ in the above equation we obtain (2.18).

Therefore, in order to complete the proof of both Theorem 1.1 and Theorem 2.3 we need to show that $N(\mathcal{R}):=\sqrt{\min I_{\mathcal{R}}}$ is a norm on $\mathcal{R}$. Since $I_{\alpha \mathcal{R}}(\alpha f)=\alpha^{2} I_{\mathcal{R}}(f)$ for every $\alpha \in \mathbb{R}$, and since we minimize over a vector space, we have

$$
N(\alpha \mathcal{R})=|\alpha| N(\mathcal{R}) .
$$

Note also that if $f_{a}$ is a minimizer of $I_{\mathcal{R}^{a}}$ for $a=1,2$, then

$$
\left(\left.\int_{B}\left|\operatorname{Sym}\left(d f_{1}+d f_{2}\right)-\frac{1}{6}\left(\mathcal{R}_{k i j l}^{1}+\mathcal{R}_{k i j l}^{2}\right) x^{k} x\right|^{2}\right|^{1 / 2} \leq \sum_{a=1}^{2}\left(\int_{B}\left|\operatorname{Sym}\left(d f_{a}\right)-\frac{1}{6} \mathcal{R}_{k i j l}^{a} x^{k} x^{l}\right|^{2}\right)^{1 / 2},\right.
$$

hence

$$
N\left(\mathcal{R}^{1}+\mathcal{R}^{2}\right) \leq N\left(\mathcal{R}^{1}\right)+N\left(\mathcal{R}^{2}\right) .
$$

Therefore $N$ is a semi-norm. A similar calculation shows that $2 I_{\mathcal{R}^{1}}\left[f_{1}\right]+2 I_{\mathcal{R}^{2}}\left[f_{2}\right]=$ $I_{\mathcal{R}^{1}+\mathcal{R}^{2}}\left[f_{1}+f_{2}\right]+I_{\mathcal{R}^{1}-\mathcal{R}^{2}}\left[f_{1}-f_{2}\right]$. This implies $f_{1} \pm f_{2}$ is a minimizer of $I_{\mathcal{R}^{1} \pm \mathcal{R}^{2}}$, so $N$ satisfies the parallelogram law. Indeed, let $f_{ \pm}$be a minimizer of $I_{\mathcal{R}^{1} \pm \mathcal{R}^{2}}$, then

$$
\begin{aligned}
2 I_{\mathcal{R}^{1}}\left[f_{1}\right]+2 I_{\mathcal{R}^{2}}\left[f_{2}\right] & =I_{\mathcal{R}^{1}+\mathcal{R}^{2}}\left[f_{1}+f_{2}\right]+I_{\mathcal{R}^{1}-\mathcal{R}^{2}}\left[f_{1}-f_{2}\right] \\
& \geq I_{\mathcal{R}^{1}+\mathcal{R}^{2}}\left[f_{+}\right]+I_{\mathcal{R}^{1}-\mathcal{R}^{2}}\left[f_{-}\right] \\
& =2 I_{\mathcal{R}^{1}}\left[\frac{f_{+}+f_{-}}{2}\right]+2 I_{\mathcal{R}^{2}}\left[\frac{f_{+}-f_{-}}{2}\right] \\
& \geq 2 I_{\mathcal{R}^{1}}\left[f_{1}\right]+2 I_{\mathcal{R}^{2}}\left[f_{2}\right] .
\end{aligned}
$$


Therefore, in order to complete the proof we need to show the positivity of $N$.

Denote $e_{i j}:=\frac{1}{6} \mathcal{R}_{k i j l} x^{k} x^{l}$. Since the minimizer of $I_{\mathcal{R}}$ exists, $N(\mathcal{R})=0$ if and only if there exists a function $f \in W^{1,2}\left(B ; \mathbb{R}^{n}\right)$ such that $(\operatorname{Sym} d f)_{i j}=e_{i j}$. The Saint-Venant lemma [Cia13, Section 6.18] implies that there exists such function if and only if

$$
\partial_{l j} e_{i k}+\partial_{k i} e_{j l}-\partial_{l i} e_{j k}-\partial_{k j} e_{i l}=0
$$

Note that

$$
\partial_{l j} e_{i k}=\frac{1}{6} \mathcal{R}_{a i k b} \partial_{l j}\left(x^{a} x^{b}\right)=\frac{1}{6} \mathcal{R}_{a i k b}\left(\delta_{a l} \delta_{b j}+\delta_{a j} \delta_{b l}\right)=\frac{1}{6}\left(\mathcal{R}_{l i k j}+\mathcal{R}_{j i k l}\right),
$$

hence (using the symmetries of the curvature tensor) we have

$$
\begin{aligned}
6\left(\partial_{l j} e_{i k}+\partial_{k i} e_{j l}-\partial_{l i} e_{j k}-\partial_{k j} e_{i l}\right) & =\mathcal{R}_{l i k j}+\mathcal{R}_{j i k l}+\mathcal{R}_{k j l i}+\mathcal{R}_{i j l k}-\mathcal{R}_{l j k i}-\mathcal{R}_{i j k l}-\mathcal{R}_{k i l j}-\mathcal{R}_{j i l k} \\
& =2\left(\mathcal{R}_{l i k j}-\mathcal{R}_{l j k i}+\mathcal{R}_{j i k l}-\mathcal{R}_{i j k l}\right) \\
& =2\left(\mathcal{R}_{l i k j}+\mathcal{R}_{l j i k}+2 \mathcal{R}_{j i k l}\right) \\
& =2\left(-\mathcal{R}_{l k j i}+2 \mathcal{R}_{j i k l}\right) \\
& =6 \mathcal{R}_{j i k l} .
\end{aligned}
$$

Therefore, the minimum energy is zero if and only if $\mathcal{R}=0$. It follows that $N(\cdot)$ is a norm on the space of Riemannian curvatures at $p$.

\section{Energy scaling for general thin elastic bodies}

In this section we prove Theorem 1.2. We begin by introducing some notations and by describing the main result of [KS14].

- Recall that $\left.T \mathcal{M}\right|_{\mathcal{S}}=T S \oplus \mathcal{N} S$, and denote by $P_{\mathcal{S}}^{\|}:\left.T \mathcal{N}\right|_{\mathcal{S}} \rightarrow T S$ and $P_{\mathcal{S}}^{\perp}:\left.T \mathcal{N}\right|_{\mathcal{S}} \rightarrow \mathcal{N} S$ the orthogonal projections. The corresponding projections of other submanifolds are defined similarly.

- We denote by $\pi_{h}: \mathcal{S}_{h} \rightarrow \mathcal{S}$, the natural projection $\pi_{h}\left(\exp _{p}(v)\right):=p$ (see (1.2)).

- We denote by $\nabla^{\mathcal{M}}$ the Levi-Civita connection on the tangent bundle of $\mathcal{M}$, and similarly for other manifolds. We denote by $\nabla^{E}$ the connection induced by the relevant Levi-Civita connection on a vector bundle $E$. For example, $\nabla^{\mathcal{N S}}$ is the connection of $\mathcal{N S}$ induced by $\nabla^{\mathcal{M}}$. We write $\nabla$ when the connection is clear from the context.

- The second fundamental form (shape operator) of $\mathcal{S}$ in $\mathcal{M}$ is defined by

$$
\mathrm{II}_{\mathcal{S}, \mathcal{M}}: T S \times \mathcal{N S} \rightarrow T \mathcal{S}, \mathrm{II}_{\mathcal{S}, \mathcal{M}}(v, \eta):=-P_{\mathcal{S}}^{\|}\left(\nabla_{v}^{\mathcal{M}} N\right),
$$

where $N$ is a local extension of $\eta$ in the normal bundle $\mathcal{N} S$. The second fundamental form of other submanifolds is defined similarly.

The main result of [KS14] is that the rescaled energies $h^{-2} E_{\mathcal{S}_{h}} \Gamma$-converge (including a compactness statement), under an appropriate notion of $W^{1,2}$ convergence, to the limit energy

$$
E_{\mathcal{S}}: W^{2,2}\left(\mathcal{S} ; \mathbb{R}^{n}\right) \times W^{1,2}\left(\mathcal{S} ; \mathcal{N} \mathcal{S}^{*} \otimes \mathbb{R}^{n}\right) \rightarrow[0, \infty]
$$


defined by

$E_{\mathcal{S}}\left(F, q^{\perp}\right):= \begin{cases}C f_{\mathcal{S}}\left(2\left|P_{\mathcal{S}}^{\|} \circ q^{-1} \circ \nabla q^{\perp}+\mathrm{II}_{\mathcal{S}, \mathcal{M}}\right|^{2}+\left|P_{\mathcal{S}}^{\perp} \circ q^{-1} \circ \nabla q^{\perp}\right|^{2}\right) d \mathrm{Vol}_{\left.\mathfrak{g}\right|_{\mathcal{S}}} & q \in \mathrm{SO}(\mathfrak{g}, \mathfrak{e}) \text { a.e. } \\ \infty & \text { otherwise, }\end{cases}$

where $q:=d F \oplus q^{\perp}$, and $C$ is some constant depending on the codimension of $\mathcal{S}$ in $\mathcal{M}$. Note that [KS14] and (3.1) uses different sign conventions for $\mathrm{II}_{\mathcal{S}, \mathcal{M}}$, which results in a sign difference in the definition of $E_{\mathcal{S}}$.

Proof (of Theorem 1.2]: It follows immediately from the main result of [KS14] described above that $\inf E_{\mathcal{S}_{h}}=O\left(h^{2}\right)$ if and only if $E_{\mathcal{S}}$ is not identically infinity, which implies that there exists $F \in W^{2,2}\left(\mathcal{S} ; \mathbb{R}^{n}\right)$ and $q^{\perp} \in W^{1,2}\left(\mathcal{S} ; \mathcal{N} \mathcal{S}^{*} \otimes \mathbb{R}^{n}\right)$ such that $d F \oplus q^{\perp} \in \mathrm{SO}(\mathfrak{g}, \mathfrak{e})$ a.e. This proves part 1 of Theorem 1.2

Furthermore, $\min E_{\mathcal{S}}=0$ if and only if $\inf E_{\mathcal{S}_{h}}=o\left(h^{2}\right)$. Note that the conditions for $E_{\mathcal{S}}\left(F, q^{\perp}\right)$ to vanish, that is $-P_{\mathcal{S}}^{\|} \circ q^{-1} \circ \nabla q^{\perp}=I_{\mathcal{S}, \mathcal{M}}$ and $P_{\mathcal{S}}^{\perp} \circ q^{-1} \circ \nabla q^{\perp}=0$, are equivalent to the condition $\nabla q^{\perp}=-d F \circ \mathrm{II}_{\mathcal{S}, \mathcal{M}}$.

We split the analysis of the case $\inf E_{\mathcal{S}_{h}}=o\left(h^{2}\right)$, that is, of $\min E_{\mathcal{S}}=0$, into several steps, details in lemmas bellows. First, we prove in Lemma 3.1 that if $\min E_{\mathcal{S}}=0$, then the minimizer is smooth, which is used throughout the rest of the proof. Next, in Lemma 3.2 we show that the condition $-P_{\mathcal{S}}^{\|} \circ q^{-1} \circ \nabla q^{\perp}=\mathrm{II}_{\mathcal{S}, \mathcal{M}}$ implies that the second form $\mathrm{II}_{F(\mathcal{S}), \mathbb{R}^{n}}$ coincides with $\mathrm{II}_{\mathcal{S}, \mathcal{M}}$ under appropriate identifications that are detailed in the lemma.

We then show, in Lemma 3.3, that the condition $P_{\mathcal{S}}^{\perp} \circ q^{-1} \circ \nabla q^{\perp}=0$ implies that the normal connection of $F(\mathcal{S})$ in $\mathbb{R}^{n}$ coincides with that of $\mathcal{S}$ in $\mathcal{M}$ (again, under appropriate identifications). Together with the identification of the second forms $\mathrm{II}_{F(\mathcal{S}), \mathbb{R}^{n}}$ and $\mathrm{II}_{\mathcal{S}, \mathcal{M}}$ (Lemma 3.2), this implies also that the covariant derivatives of the second fundamental forms coincide (Lemma 3.4). Using this and the Gauss-Codazzi-Ricci equations, we conclude in Proposition 3.5 that $\min E_{\mathcal{S}}=0$ implies $\mathcal{R}^{\mathcal{M}}(X, Y)=0$ for every $X, Y \in T \mathcal{S}$, and that for simply connected manifolds the converse also holds. This completes the proof of part 3 of Theorem 1.2 .

The smoothness of the minimizer $\left(F, q^{\perp}\right)$ of $E_{\mathcal{S}}$ in our case immediately shows that its recovery sequence $u_{h} \in W^{1,2}\left(\mathcal{S}_{h} ; \mathbb{R}^{n}\right)$, as described in [KS14, Section 6], satisfies $E_{S_{h}}\left(u_{h}\right)<C h^{4}$, which proves (1.7). This is the content of Lemma 3.6. This completes the proof of part 2 of Theorem 1.2 .

Finally, in Lemma 3.8 we prove the bound (1.8). The rest of part 4 of Theorem 1.2 immediately follows from that bound. Indeed, assume that (1.9) holds, and $\mathcal{R}^{\mathcal{N}}$ is parallel along a foliation of curves emanating from $\mathcal{S}$. Because of (1.8), assumption (1.9) implies that $\left.\mathcal{R}^{\mathcal{M}}\right|_{\mathcal{S}} \equiv 0$ and the parallelism of $\mathcal{R}$ then implies that $\left.\mathcal{R}^{\mathcal{M}}\right|_{S_{h}} \equiv 0$. If $\mathcal{S}$ is simply-connected, then also $\mathcal{S}_{h}$, since they are homotopy equivalent for small enough $h$. A simply-connected $n$-dimensional manifold with zero curvature can be isometrically immersed in $\mathbb{R}^{n}$ [Cia05, Theorem 1.6-1]. Thus, $\min E_{\delta_{h}}=0$ (since we do not impose any boundary conditions or external forces).

\subsection{Proofs regarding the scaling inf $E_{\mathcal{S}_{h}}=o\left(h^{2}\right)$}

In this section we prove our results concerning the scaling inf $E_{\mathcal{S}_{h}}=o\left(h^{2}\right)$. These include most of part 2 and part 3 of Theorem 1.2. (1.7) in part 2, and part 4 of the theorem are proved in Section 3.2 
Lemma 3.1 If $\min E_{\mathcal{S}}=0$, then the minimizer $\left(F, q^{\perp}\right)$ is smooth and unique up to rigid motions.

Proof: We use the following notations: the indices $i, j, \ldots$ are in the range $1 . . k$, the indices $a, b, \ldots$ are in $k+1, \ldots, n$, and the indices $I, J, \ldots$ are in $1 . . n$.

Choose local coordinates $x^{i}$ on $\mathcal{S}$ and a frame $v_{a}$ for $\mathcal{N} \mathcal{S}$. We extend the coordinate system to a tubular neighborhood by choosing $x^{a}$, such that $\left.\partial_{a}\right|_{s}=v_{a}$. Therefore, $\mathfrak{g}_{i a}=0$ along $\mathcal{S}$. In these coordinates write $q_{a}^{\perp}=q^{\perp}\left(\partial_{a}\right)$. Let $\Gamma_{I J}^{K}$ be the Christoffel symbols of $(\mathcal{M}, \mathfrak{g})$ along $\mathcal{S}$. They are smooth functions of $x^{i}$.

Let $F \in W_{\text {iso }}^{2,2}\left(\mathcal{S} ; \mathbb{R}^{n}\right)$ and $q^{\perp} \in W^{1,2}\left(\mathcal{S} ; N^{*} \mathcal{S} \otimes \mathbb{R}^{n}\right)$ satisfy $E_{\mathcal{S}}\left(F, q^{\perp}\right)=0$. This implies the following

1.

$$
d F \oplus q^{\perp} \in \mathrm{SO}(\mathfrak{g}, \mathfrak{e}) \text { almost everywhere. }
$$

2. For every $X \in T S$ and $\eta \in \mathcal{N} S$,

$$
-P_{\mathcal{S}}^{\|} \circ q^{-1}\left(\left(\nabla_{X}^{\mathcal{N S} \otimes \mathbb{R}^{n}} q^{\perp}\right)(\eta)\right)=\mathrm{II}_{\delta, \mathcal{M}}(X, \eta) .
$$

3. For every $X \in T S$ and $\eta \in \mathcal{N} S$,

$$
P_{\mathcal{S}}^{\perp} \circ q^{-1}\left(\left(\nabla_{X}^{\mathcal{N S} \mathcal{S}^{*} \otimes \mathbb{R}^{n}} q^{\perp}\right)(\eta)\right)=0
$$

Condition 1 implies that $\partial_{i} F \cdot \partial_{j} F=\mathfrak{g}_{i j}, \partial_{i} F \cdot q_{a}^{\perp}=0$ and $q_{a}^{\perp} \cdot q_{b}^{\perp}=\mathfrak{g}_{a b}$, where $\cdot$ stands for the standard inner-product in $\mathbb{R}^{n}$.

Since $\left\{\partial_{k} F\right\} \cup\left\{q_{a}^{\perp}\right\}$ is a basis to $\mathbb{R}^{n}$, we can write

$$
\partial_{i} \partial_{j} F=A_{i j}^{l} \partial_{l} F+A_{i j}^{a} q_{a}^{\perp}
$$

for some functions $A_{i j}^{l}, A_{i j}^{a}$.

We now show that $A_{i j}^{l}=\Gamma_{i j}^{l}$ by repeating the calculation of the expression for the Christoffel symbols of the Levi-Civita connection on $\mathcal{S}$. Note that all the arguments below are valid in this Sobolev regularity, as they rely only on the validity of the product rule and on $\partial_{i} \partial_{j}=\partial_{j} \partial_{i}$, both of them hold in this regularity.

$$
\begin{aligned}
\partial_{i} \partial_{j} F \cdot \partial_{l} F & =\partial_{i} \mathfrak{g}_{j l}-\partial_{j} F \cdot \partial_{i} \partial_{l} F \\
& =\partial_{i} \mathfrak{g}_{j l}-\partial_{l} \mathfrak{g}_{i j}+\partial_{l} \partial_{j} F \cdot \partial_{i} F \\
& =\partial_{i} \mathfrak{g}_{j l}-\partial_{l} \mathfrak{g}_{i j}+\partial_{j} \mathfrak{g}_{l i}-\partial_{l} F \cdot \partial_{j} \partial_{i} F,
\end{aligned}
$$

and therefore

$$
A_{i j}^{m} \mathfrak{g}_{m l}=\partial_{i} \partial_{j} F \cdot \partial_{l} F=\frac{1}{2}\left(\partial_{i} \mathfrak{g}_{j l}-\partial_{l} \mathfrak{g}_{i j}+\partial_{j} \mathfrak{g}_{l i}\right)=\Gamma_{i j}^{m} \mathfrak{g}_{m l} .
$$

Up to now we have

$$
\partial_{i} \partial_{j} F=\Gamma_{i j}^{l} \partial_{l} F+A_{i j}^{a} q_{a}^{\perp} .
$$

Next, we consider conditions 2 and 3. By definition,

$$
\begin{aligned}
\left(\nabla_{\partial_{i}}^{\mathcal{N S} \otimes \mathbb{R}^{n}} q^{\perp}\right)\left(\partial_{a}\right) & =\partial_{i}\left(q^{\perp}\left(\partial_{a}\right)\right)-q^{\perp}\left(\nabla_{\partial_{i}}^{\mathcal{N S}} \partial_{a}\right) \\
& =\partial_{i} q_{a}^{\perp}-q^{\perp}\left(\Gamma_{i a}^{b} \partial_{b}\right) \\
& =\partial_{i} q_{a}^{\perp}-\Gamma_{i a}^{b} q_{b}^{\perp}
\end{aligned}
$$


where in the second line we used

$$
\nabla_{\partial_{i}}^{\mathcal{N S}} \partial_{a}=P^{\perp}\left(\nabla_{\partial_{i}}^{\mathcal{N}} \partial_{a}\right)=P^{\perp}\left(\Gamma_{i a}^{j} \partial_{j}+\Gamma_{i a}^{b} \partial_{b}\right)=\Gamma_{i a}^{b} \partial_{b} .
$$

By (3.3) and (3.6) together with the identity $P_{\mathcal{S}}^{\|} \circ q^{-1}=q^{-1} \circ P_{F(\mathcal{S})^{\prime}}^{\|}$, we get

$$
\begin{aligned}
P_{F(\mathcal{S})}^{\|}\left(\partial_{i} q_{a}^{\perp}\right) & =-d F \circ \mathrm{II}_{\mathcal{S}, \mathcal{M}}\left(\partial_{i}, \partial_{a}\right):=d F \circ P_{\mathcal{S}}^{\|}\left(\nabla_{\partial_{i}}^{\mathcal{M}} \partial_{a}\right) \\
& =d F \circ P_{\mathcal{S}}^{\|}\left(\Gamma_{i a}^{j} \partial_{j}+\Gamma_{i a}^{b} \partial_{b}\right) \\
& =\Gamma_{i a}^{j} \partial_{j} F .
\end{aligned}
$$

Now, equations (3.4) and (3.6) yield

$$
0=P_{F(\mathcal{S})}^{\perp}\left(\partial_{i} q_{a}^{\perp}-\Gamma_{i a}^{b} q_{b}^{\perp}\right)=P_{F(\mathcal{S})}^{\perp}\left(\partial_{i} q_{a}^{\perp}\right)-\Gamma_{i a}^{b} q_{b}^{\perp} .
$$

Combining (3.7) and (3.8) we obtain

$$
\partial_{i} q_{a}^{\perp}=P_{F(\mathcal{S})}^{\|}\left(\partial_{i} q_{a}^{\perp}\right)+P_{F(\mathcal{S})}^{\perp}\left(\partial_{i} q_{a}^{\perp}\right)=\Gamma_{i a}^{j} \partial_{j} F+\Gamma_{i a}^{b} q_{b}^{\perp} .
$$

Using (3.9) we have

$$
\partial_{i} \partial_{j} F \cdot q_{a}^{\perp}=\partial_{i} \mathfrak{g}_{j a}-\partial_{j} F \cdot \partial_{i} q_{a}^{\perp}=-\Gamma_{i a}^{l} \mathfrak{g}_{l j}
$$

hence in (3.5) the coefficients $A_{i j}^{a}$ satisfy

$$
A_{i j}^{a}=-\Gamma_{i b}^{l} \mathfrak{g}_{l j} \mathfrak{g}^{a b}
$$

Therefore the equation for $\partial_{i} \partial_{j} F$ is

$$
\partial_{i} \partial_{j} F=\Gamma_{i j}^{l} \partial_{l} F-\Gamma_{i b}^{l} \mathfrak{g}_{l j} \mathfrak{g}^{a b} q_{a}^{\perp} .
$$

Since $\mathfrak{g}_{i j}, \mathrm{~g}^{a b}$ and $\Gamma_{I J}^{K}$ are smooth functions, (3.9) and (3.10) show that $F$ and $q^{\perp}$ are actually smooth, by a bootstrap argument.

Given the smoothness, the uniqueness follows from [Ten71, Section 3], as explained in the proof of Lemma 3.5 below.

Next, we prove several lemmas leading to the proof of Proposition 3.5. In the next two lemmas, we give a geometric interpretation of what does it mean for a pair $\left(F, q^{\perp}\right)$ to satisfy $E_{\mathcal{S}}\left(F, q^{\perp}\right)=0$. Recall that $E_{\mathcal{S}}\left(F, q^{\perp}\right)=0$ if and only if $q:=d F \oplus q^{\perp} \in \mathrm{SO}(\mathfrak{g}, \mathfrak{e})$, and (3.3) and (3.4) hold, i.e. $-P_{\mathcal{S}}^{\|} \circ q^{-1} \circ \nabla q^{\perp}=\mathrm{II}_{\mathcal{S}, \mathcal{M}}$ and $P_{\mathcal{S}}^{\perp} \circ q^{-1} \circ \nabla q^{\perp}=0$.

In these lemmas we will repeatedly identify $\mathcal{S}$ and $F(\mathcal{S})$, and therefore we can view $f: \mathcal{S} \rightarrow \mathbb{R}$ as a function on $F(\mathcal{S})$. Under this identification $X(f)=d F(X)(f)$ for every $X \in T \mathcal{S}$, where in the right-hand side we consider $f$ as a function $F(\mathcal{S}) \rightarrow \mathbb{R}$. This identification also extends to the trivial bundles $\mathcal{S} \times \mathbb{R}^{n}$ and $\left.T \mathbb{R}^{n}\right|_{F(\mathcal{S})}=F(\mathcal{S}) \times \mathbb{R}^{n}$. Slightly abusing notation, we will denote the (trivial) connections on both bundles by $\nabla^{\mathbb{R}^{n}}$. The identification $X(f)=d F(X)(f)$ extends (entry-wise) to $\nabla^{\mathbb{R}^{n}}$; namely, for $f: \mathcal{S} \rightarrow \mathbb{R}^{n}$ and $X \in T \mathcal{S}, \nabla_{X}^{\mathbb{R}^{n}} f=\nabla_{d F(X)}^{\mathbb{R}^{n}} f$, where in the right-hand side $f$ is considered as a section of $\left.T \mathbb{R}^{n}\right|_{F(\mathcal{S})}$.

Lemma 3.2 (Equality of second fundamental forms) Assume $q \in S O(\mathfrak{g}, \mathfrak{e}) . \quad I I_{s, \mathfrak{M}}=$ $-P_{\mathcal{S}}^{\|} \circ q^{-1} \circ \nabla q^{\perp}$ holds if and only if

$$
d F\left(I I_{\mathcal{S}, M(}(X, \eta)\right)=I I_{F(\mathcal{S}), \mathbb{R}^{n}}\left(d F(X), q^{\perp}(\eta)\right) \text { for every }(X, \eta) \in T \mathcal{S} \times \mathcal{N S} .
$$


This lemma shows that $\mathrm{II}_{F(\mathcal{S}), \mathbb{R}^{n}}$ and $\mathrm{II}_{\mathcal{S}, \mathcal{M}}$ coincide, when we identify $T \mathcal{S} \cong d F(T \mathcal{S}), \mathcal{N} \mathcal{S} \cong$ $\mathcal{N} F(\mathcal{S})$ using the maps $d F$ and $q^{\perp}$, respectively. Here $\mathcal{N} F(\mathcal{S}):=(d F(T \mathcal{S}))^{\perp}$ is the normal bundle to the image $F(S)$ in $\mathbb{R}^{n}$.

Proof: Let $(X, \eta) \in T_{p} \mathcal{S} \times \mathcal{N}_{p} \mathcal{S}$ and let $N$ be a local extension of $\eta$ normal to $\mathcal{S}$. Then, identifying the trivial bundle $\mathcal{S} \times \mathbb{R}^{n}$ with $\left.T \mathbb{R}^{n}\right|_{F(\mathcal{S})}$, and using the identity $P_{\mathcal{S}}^{\|} \circ q^{-1}=$ $q^{-1} \circ P_{F(\mathcal{S})}^{\|}$(which holds since $\left.q \in \mathrm{SO}(\mathfrak{g}, \mathrm{e})\right)$, we have

$$
\begin{aligned}
d F_{p} \circ P_{\mathcal{S}}^{\|} \circ q^{-1}\left(\left(\nabla_{X}^{\mathcal{N S} \mathcal{S}^{*} \otimes \mathbb{R}^{n}} q^{\perp}\right)(\eta)\right) & =P_{F(\mathcal{S})}^{\|}\left(\left(\nabla_{X}^{\mathcal{N S} \mathcal{S}^{*} \otimes \mathbb{R}^{n}} q^{\perp}\right)(\eta)\right) \\
& =P_{F(\mathcal{S})}^{\|}\left(\nabla_{X}^{\mathbb{R}^{n}}\left(q^{\perp}(N)\right)-q^{\perp}\left(\nabla_{X}^{\mathcal{N S}} N\right)\right) \\
& =P_{F(\mathcal{S})}^{\|} \nabla_{X}^{\mathbb{R}^{n}}\left(q^{\perp}(N)\right),
\end{aligned}
$$

Hence $\mathrm{II}_{\mathcal{S}, \mathcal{M}}=-P_{\mathcal{S}}^{\|} \circ q^{-1} \circ \nabla q^{\perp}$ is equivalent to

$$
d F_{p}\left(\mathrm{II}_{\mathcal{S}, \mathcal{M}}(X, \eta)\right)=-P_{F(\mathcal{S})}^{\|}\left(\nabla_{d F_{p}(X)}^{\mathbb{R}^{n}}\left(q^{\perp}(N)\right)\right) .
$$

On the other hand, the right-hand side of this equality is the definition of $\operatorname{II}_{F(\mathcal{S}), \mathbb{R}^{n}}\left(d F_{p}(X), q^{\perp}(\eta)\right)$. Therefore, we obtain,

$$
\mathrm{II}_{\mathcal{S}, \mathcal{M}}=-P_{\mathcal{S}}^{\|} \circ q^{-1} \circ \nabla q^{\perp} \Longleftrightarrow d F\left(\mathrm{II}_{\mathcal{S}, \mathcal{M}}(X, \eta)\right)=\mathrm{II}_{F(\mathcal{S}), \mathbb{R}^{n}}\left(d F_{p}(X), q^{\perp}(\eta)\right) .
$$

Lemma 3.3 (Equality of normal connections) Let $F, q^{\perp}$ be smooth, and $q \in S O(\mathfrak{g}, \mathfrak{e})$. Then $P_{\mathcal{S}}^{\perp} \circ q^{-1} \circ \nabla q^{\perp}=0$ holds if and only if

$$
q^{\perp}\left(\nabla_{X}^{\mathcal{N S}} \sigma\right)=\nabla_{d F(X)}^{N F(\mathcal{S})}\left(q^{\perp} \sigma\right) \text { for every } X \in T \mathcal{S} \text { and } \sigma \in \Gamma(\mathcal{N S}),
$$

where $\nabla_{d F(X)}^{N F(\mathcal{S})}\left(q^{\perp} \sigma\right)=P_{F(\mathcal{S})}^{\perp}\left(\nabla_{X}^{\mathbb{R}^{n}}\left(q^{\perp}(\sigma)\right)\right)$.

This lemma shows that the normal connections $\nabla^{\mathcal{N S}}$ and $\nabla^{N F(S)}$ coincide, under the identifications $T \mathcal{S} \cong d F(T \mathcal{S}), \mathcal{N S} \cong N F(S)$ induced by the maps $d F$ and $q^{\perp}$, respectively.

Proof: Given $X \in T \mathcal{S}$ and $\sigma \in \Gamma(\mathcal{N S})$ we have

$$
\nabla_{X}^{\mathbb{R}^{n}}\left(q^{\perp}(\sigma)\right)=\left(\nabla_{X}^{\mathcal{N} S \otimes \mathbb{R}^{n}} q^{\perp}\right)(\sigma)+q^{\perp}\left(\nabla_{X}^{\mathcal{N S} \sigma}\right),
$$

so

$$
P_{\mathcal{S}}^{\perp} \circ q^{-1}\left(\nabla_{X}^{\mathbb{R}^{n}}\left(q^{\perp}(\sigma)\right)\right)=P_{\mathcal{S}}^{\perp} \circ q^{-1}\left(\left(\nabla_{X}^{\mathcal{N} S \otimes \mathbb{R}^{n}} q^{\perp}\right)(\sigma)\right)+\nabla_{X}^{\mathcal{N S}} \sigma .
$$

Thus, $P_{\mathcal{S}}^{\perp} \circ q^{-1} \circ \nabla q^{\perp}=0$ holds if and only if

$$
P_{\mathcal{S}}^{\perp} \circ q^{-1}\left(\nabla_{X}^{\mathbb{R}^{n}}\left(q^{\perp}(\sigma)\right)\right)=\nabla_{X}^{\mathcal{N S}}
$$

Using $q^{\perp} \circ P_{\mathcal{S}}^{\perp} \circ q^{-1}=P_{F(\mathcal{S})}^{\perp}$ (which holds since $q \in \mathrm{SO}(\mathfrak{g}, \mathrm{e})$ ), we have that the above equation holds if and only if

$$
P_{F(\mathcal{S})}^{\perp}\left(\nabla_{X}^{\mathbb{R}^{n}}\left(q^{\perp}(\sigma)\right)\right)=q^{\perp}\left(\nabla_{X}^{\mathcal{N S}} \sigma\right) .
$$


Next, we prove the final lemma required for establishing Proposition 3.5. This lemma combines the previous two lemmas, 3.2 and 3.3 and shows that the derivatives of the second fundamental forms coincide (again under the appropriate identifications).

In this lemma, we will use the following notation: $B_{\mathcal{S}}: T \mathcal{S} \times T \mathcal{S} \rightarrow \mathcal{N S}$ is defined by $\left\langle B_{\mathcal{S}}(X, Y), \eta\right\rangle=\left\langle\mathrm{II}_{\mathcal{S}, \mathcal{M}}(X, \eta), Y\right\rangle$. We also consider $B_{\mathcal{S}}$ as a map $T \mathcal{S} \times T \mathcal{S} \times \mathcal{N} \mathcal{S} \rightarrow \mathbb{R}$, via $(X, Y, \eta) \mapsto\left\langle B_{\mathcal{S}}(X, Y), \eta\right\rangle$. Finally, we extend the covariant derivative to tensors of this type in the usual way, as follows:

$$
\nabla_{X}^{\mathcal{N}} B_{\mathcal{S}}(Y, Z, \eta)=X\left(B_{\mathcal{S}}(Y, Z, \eta)\right)-B_{\mathcal{S}}\left(\nabla_{X}^{\mathcal{S}} Y, Z, \eta\right)-B_{\mathcal{S}}\left(Y, \nabla_{X}^{\mathcal{S}} Z, \eta\right)-B_{\mathcal{S}}\left(Y, Z, \nabla_{X}^{\mathcal{N S}} \eta\right) .
$$

Lemma 3.4 (Coincidence of the derivatives) Let $\left(F, q^{\perp}\right)$ satisfy (3.2)-(3.4). Then, for every $X, Y \in \Gamma(T S)$ and $\eta \in \Gamma(\mathcal{N S})$ the following hold:

1 .

$$
q^{\perp}\left(B_{\mathcal{S}}(X, Y)\right)=B_{F(\mathcal{S})}(d F(X), d F(Y))
$$

2.

$$
B_{\mathcal{S}}(X, Y, \eta)=B_{F(\mathcal{S})}\left(d F(X), d F(Y), q^{\perp}(\eta)\right)
$$

3.

$$
\nabla_{X}^{\mathcal{M}} B_{\mathcal{S}}(Y, Z, \eta)=\nabla_{d F(X)}^{\mathbb{R}^{n}} B_{F(\mathcal{S})}\left(d F(Y), d F(Z), q^{\perp}(\eta)\right)
$$

Proof: Lemma3.2, together with the fact that $F: \mathcal{S} \rightarrow F(\mathcal{S})$ is an isometry, implies

$$
\begin{aligned}
\left\langle B_{\mathcal{S}}(X, Y), \eta\right\rangle & =\left\langle\mathrm{II}_{\mathcal{S}, \mathcal{M}}(X, \eta), Y\right\rangle=\left\langle d F\left(\mathrm{II}_{\mathcal{S}, \mathcal{M}}(X, \eta)\right), d F(Y)\right\rangle \\
& =\left\langle\mathrm{II}_{F(\mathcal{S}), \mathbb{R}^{n}}\left(d F(X), q^{\perp}(\eta)\right), d F(Y)\right\rangle \\
& =\left\langle B_{F(\mathcal{S})}(d F(X), d F(Y)), q^{\perp}(\eta)\right\rangle .
\end{aligned}
$$

Since $q^{\perp}: \mathcal{N S} \rightarrow \mathcal{N} F(\mathcal{S})$ is an isometry,

$$
\left\langle B_{\mathcal{S}}(X, Y), \eta\right\rangle=\left\langle q^{\perp}\left(B_{\mathcal{S}}(X, Y)\right), q^{\perp}(\eta)\right\rangle .
$$

Combining (3.14) and (3.15) proves (3.11) and (3.12).

We now prove (3.13). Using (3.12), we get

$$
\begin{aligned}
\nabla_{X}^{\mathcal{M}} B_{\mathcal{S}}(Y, Z, \eta)= & X\left(B_{F(\mathcal{S})}\left(d F(Y), d F(Z), q^{\perp}(\eta)\right)\right)-B_{F(\mathcal{S})}\left(d F\left(\nabla_{X}^{\mathcal{S}} Y\right), d F(Z), q^{\perp}(\eta)\right) \\
& -B_{F(\mathcal{S})}\left(d F(Y), d F\left(\nabla_{X}^{\mathcal{S}} Z\right), q^{\perp}(\eta)\right)-B_{F(\mathcal{S})}\left(d F(Y), d F(Z), q^{\perp}\left(\nabla_{X}^{\mathcal{N} S} \eta\right)\right) .
\end{aligned}
$$

On the other hand,

$$
\begin{aligned}
\nabla_{d F(X)}^{\mathbb{R}^{n}} B_{F(\mathcal{S})}\left(d F(Y), d F(Z), q^{\perp}(\eta)\right)= & d F(X)\left(B_{F(\mathcal{S})}\left(d F(Y), d F(Z), q^{\perp}(\eta)\right)\right)-B_{F(\mathcal{S})}\left(\nabla_{d F(X)}^{F(\mathcal{S})} d F(Y), d F(Z), q^{\perp}(\eta)\right) \\
& -B_{F(\mathcal{S})}\left(d F(Y), \nabla_{d F(X)}^{F(\mathcal{S})} d F(Z), q^{\perp}(\eta)\right)-B_{F(\mathcal{S})}\left(d F(Y), d F(Z),\left(\nabla_{d F(X)}^{N F(\mathcal{S})}\left(q^{\perp} \eta\right)\right) .\right.
\end{aligned}
$$

The first summand is the same by the identification of $\mathcal{S}$ and $F(\mathcal{S})$ discussed before Lemma 3.2. The second summand is the same since $d F\left(\nabla_{X}^{\mathcal{S}} Y\right)=\nabla_{d F(X)}^{F(\mathcal{S})} d F(Y)$ because $F: S \rightarrow F(S)$ is an isometry, hence preserves the connection. The last two summands are the same by Lemma 3.3 .

Finally, we use the above to prove part 3 of Theorem 1.2 
Proposition 3.5 Let $\mathcal{R}^{\mathcal{N}}$ the Riemannian curvature tensor of $\mathcal{M}$. Assume there exists $F$ and $q^{\perp}$ that satisfy equations (3.2)-(3.4), then

$$
\mathcal{R}^{\mathcal{M}}(X, Y)=0 \quad \forall X, Y \in T \mathcal{S} .
$$

If $\mathcal{S}$ is simply connected, then the converse holds. Moreover, $F$ and $q^{\perp}$ are unique up to a rigid motion.

Proof: In this proof, $X, Y, Z, T \in T \mathcal{S}$, and $\eta, \zeta \in N \mathcal{S}$. First assume the existence of such $F, q^{\perp}$. The Gauss equation [dC92, Chapter 6, Proposition 3.1], together with (3.11) and the fact that $d F \oplus q^{\perp}$ is an isometry, imply

$$
\begin{aligned}
\left\langle\mathcal{R}^{\mathcal{M}}\right. & (X, Y) Z, T\rangle=\left\langle R^{\mathcal{S}}(X, Y) Z, T\right\rangle-\left\langle B_{\mathcal{S}}(Y, T), B_{\mathcal{S}}(X, Z)\right\rangle+\left\langle B_{\mathcal{S}}(X, T), B_{\mathcal{S}}(Y, Z)\right\rangle \\
= & \left\langle\mathcal{R}^{F(\mathcal{S})}(d F(X), d F(Y)) d F(Z), d F(T)\right\rangle-\left\langle B_{F(\mathcal{S})}(d F(Y), d F(T)), B_{F(\mathcal{S})}(d F(X), d F(Z))\right\rangle \\
& +\left\langle B_{F(\mathcal{S})}(d F(X), d F(T)), B_{F(\mathcal{S})}(d F(Y), d F(Z))\right\rangle \\
= & \left\langle\mathcal{R}^{\mathbb{R}^{n}}(d F(X), d F(Y)) d F(Z), d F(T)\right\rangle=0 .
\end{aligned}
$$

Applying the Coddazi equation [dC92, Chapter 6, Proposition 3.4], and using (3.13) we have that

$$
\begin{aligned}
\left\langle\mathcal{R}^{\mathcal{M}}(X, Y) Z, \eta\right\rangle & =\nabla_{Y}^{\mathcal{M}} B_{\mathcal{S}}(X, Z, \eta)-\nabla_{X}^{\mathcal{M}} B_{\mathcal{S}}(Y, Z, \eta) \\
& =\nabla_{d F(Y)}^{\mathbb{R}^{n}} B_{F(\mathcal{S})}\left(d F(X), d F(Z), q^{\perp}(\eta)\right)-\nabla_{d F(X)}^{\mathbb{R}^{n}} B_{F(\mathcal{S})}\left(d F(Y), d F(Z), q^{\perp}(\eta)\right) \\
& =\left\langle\mathcal{R}^{\mathbb{R}^{n}}(d F(X), d F(Y)) d F(Z), q^{\perp}(\eta)\right\rangle=0 .
\end{aligned}
$$

(3.16) and (3.17) together imply

$$
\mathcal{R}^{\mathcal{M}}(X, Y) Z=0
$$

Finally, the equality of the normal connections (Lemma 3.3) implies equality of the normal curvatures(i.e. the curvature tensors associated with the normal connections)

$$
\left\langle\mathcal{R}_{\mathcal{S}}^{\perp}(X, Y) \eta, \zeta\right\rangle=\left\langle\mathcal{R}_{F(\mathcal{S})}^{\perp}(d F(X), d F(Y)) q^{\perp}(\eta), q^{\perp}(\zeta)\right\rangle,
$$

and therefore, using Ricci equation [dC92, Chapter 6, Proposition 3.1], we have

$$
\begin{aligned}
& \left\langle\mathcal{R}^{\mathcal{M}}(X, Y) \eta, \zeta\right\rangle=\left\langle\left[\mathrm{II}_{\mathcal{S}, \mathcal{M}}(X, \eta), \mathrm{II}_{\mathcal{S}, \mathcal{M}}(X, \zeta)\right], Y\right\rangle+\left\langle\mathcal{R}_{\mathcal{S}}^{\perp}(X, Y) \eta, \zeta\right\rangle \\
& =\left\langle\left[\operatorname{II}_{F(\mathcal{S}), \mathbb{R}^{n}}\left(d F(X), q^{\perp}(\eta)\right), \mathrm{II}_{F(\mathcal{S}), \mathbb{R}^{n}}\left(d F(X), q^{\perp}(\zeta)\right)\right], d F(Y)\right\rangle+\left\langle\mathcal{R}_{F(\mathcal{S})}^{\perp}(d F(X), d F(Y)) q^{\perp}(\eta), q^{\perp}(\zeta)\right\rangle \\
& =\left\langle\mathcal{R}^{\mathbb{R}^{n}}(d F(X), d F(Y)) q^{\perp} \eta, q^{\perp} \zeta\right\rangle=0 .
\end{aligned}
$$

The Codazzi equation (3.17), and the symmetries of $\mathbb{R}^{\mathcal{M}}$ also imply that

$$
\left\langle\mathcal{R}^{\mathcal{M}}(X, Y) \eta, Z\right\rangle=-\left\langle\mathcal{R}^{\mathcal{M}}(X, Y) Z, \eta\right\rangle=0,
$$

and therefore

$$
\mathcal{R}^{\mathcal{M}}(X, Y) \eta=0 \text {. }
$$

Together with (3.18), this implies that

$$
\mathcal{R}^{\mathcal{N}}(X, Y)=0
$$


Now assume $\mathcal{R}^{\mathcal{M}}(X, Y)=0$. Then $\mathrm{II}_{\mathcal{S}, \mathcal{M}}$ and $\nabla^{\perp}$ satisfy the Gauss-Ricci-Codazzi equations with zero left-hand side, hence by [Ten71, Section 3] there exist, locally, smooth $F, q^{\perp}$ as required, and are unique up to a rigid motion 3 Finally, if $\mathcal{S}$ is simply connected, then $F$ and $q^{\perp}$ can be chosen on whole $\mathcal{S}$ (see remark at the end of [Ten71], or [Che00, Section 3.2]).

\subsection{Proofs regarding the scaling inf $E_{S_{h}}=O\left(h^{4}\right)$}

In this section we prove the results concerning the $h^{4}$ energy scaling; namely, that $\inf E_{\delta_{h}}=o\left(h^{2}\right)$ implies inf $E_{\mathcal{S}_{h}}=O\left(h^{4}\right)$ (thus completing the proof of part 2 of Theorem 1.2) and that inf $h^{-4} E_{\mathcal{S}_{h}}$ is bounded from below by an integral of the curvature along $\mathcal{S}$ (part 4 of Theorem 1.2).

Lemma 3.6 If $\inf E_{\mathcal{S}_{h}}=o\left(h^{2}\right)$, then there exists a sequence of maps $u_{h} \in W^{1,2}\left(\mathcal{S}_{h} ; \mathbb{R}^{n}\right)$ such that $E_{S_{h}}\left[u_{h}\right]<C h^{4}$ for some constant $C>0$ depending on $(\mathcal{M}, \mathfrak{g})$.

Proof: This follows from the analysis in [KS14, Proposition 6.3]. Indeed, inf $E_{\mathcal{S}_{h}}=o\left(h^{2}\right)$ implies $\min E_{\mathcal{S}}=0$. Therefore, by Lemma 3.1, there exists smooth $F: \mathcal{S} \rightarrow \mathbb{R}^{n}$ and $q^{\perp}: S \rightarrow \mathcal{N} \mathcal{S}^{*} \otimes \mathbb{R}^{n}$ such that $d F \oplus q^{\perp} \in \mathrm{SO}(\mathfrak{g}, \mathfrak{e})$ and $\nabla q^{\perp}=-d F \circ \mathrm{II}_{\delta, \mathcal{M}}$.

Using the coordinates and index conventions of Lemma 3.1 define $u_{h}\left(x^{i}, x^{a}\right)=F\left(x^{i}\right)+$ $q^{\perp}\left(x^{i}, x^{a} \partial_{a}\right)$ (this is the coordinate equivalent of the recovery sequence [KS14, Equation (6.1)]). The analysis in the proof of [KS14, Proposition 6.3] implies that

$$
\operatorname{dist}\left(d u_{h}, \mathrm{SO}(\mathfrak{g}, \mathfrak{e})\right)=\left(\left|\nabla q^{\perp}\right|+\left|d u_{h}\right|\right) O\left(h^{2}\right)=O\left(h^{2}\right),
$$

where the second equality follows from the fact that $q^{\perp}$ and $u_{h}$ are uniformly bounded in $C^{1}$. Therefore,

$$
E_{\mathcal{S}_{h}}\left[u_{h}\right]=\int_{\mathcal{S}_{h}} \operatorname{dist}^{2}\left(d u_{h}, \mathrm{SO}(\mathfrak{g}, \mathfrak{e})\right) d \operatorname{Vol}_{\mathfrak{g}} \leq \int_{\mathcal{S}_{h}} C h^{4} d \mathrm{Vol}_{\mathfrak{g}}=C h^{4}
$$

For the proof of Lemma 3.8 below, we need the following immediate corollary of Theorem 1.1.

Corollary 3.7 Let $K \subset \mathcal{M}$ be compact. Then

$$
\lim _{h \rightarrow 0} \sup _{q \in K} \inf _{u \in W^{1,2}\left(B_{h}(q) ; \mathbb{R}^{n}\right)} \frac{1}{h^{4}} E_{B_{h}(q)}[u]-\left|\mathcal{R}_{q}\right|^{2} \mid=0 .
$$

Proof: Assume, for the sake of contradiction, that (3.19) does not hold. Then there exist $\varepsilon>0$ and a sequence $h_{i} \rightarrow 0$ and $p_{i} \in K$ such that for every $i$,

$$
\left.\left|\inf \frac{1}{h_{i}^{4}} E_{B_{h_{i}}\left(p_{i}\right)}-\right| \mathcal{R}_{p_{i}}\right|^{2} \mid>\varepsilon .
$$

Since $K$ is compact, we can assume that $p_{i} \rightarrow p \in K$. This contradicts Theorem 2.3, since $\left|\mathcal{R}_{p_{i}}\right| \rightarrow\left|\mathcal{R}_{p}\right|$.

\footnotetext{
${ }^{3}$ The main theorem in [Ten71] only states the uniqueness of $F$, however its proof (specifically, the last paragraph on p. 34) shows the uniqueness of $q^{\perp}$ as well.
} 
Lemma 3.8

$$
\liminf _{h \rightarrow 0}\left(\inf h^{-4} E_{\mathcal{S}_{h}}\right) \geq c \int_{S}\left|\mathcal{R}^{\mathcal{M}}\right|^{2} d V o l_{\left.\mathfrak{g}\right|_{\mathcal{S}}}
$$

where $|\cdot|$ is the norm defined in Theorem 1.1 and $c$ is a universal constant.

Proof: First, we recall that the map $p \mapsto\left|\mathcal{R}_{p}^{\mathcal{M}}\right|$ is continuous. Fix $\varepsilon>0$, and let $\left\{V^{i}\right\}_{i=1}^{m}$ be a partition of $\mathcal{S}$ into small regulars sets (e.g. embedded regular simplices) such that

$$
\frac{1}{\operatorname{Vol}_{\left.\mathfrak{g}\right|_{\mathcal{S}}}(\mathcal{S})} \sum_{i}^{m} \operatorname{Vol}_{\left.\mathfrak{g}\right|_{\mathcal{S}}}\left(V^{i}\right)\left|\mathcal{R}_{p_{i}}^{\mathcal{M}}\right|^{2}>f_{S}\left|\mathcal{R}^{\mathcal{M}}\right|^{2} d \operatorname{Vol}_{\left.\mathfrak{g}\right|_{S}}-\varepsilon
$$

for some $p_{i} \in V^{i}$. We can, furthermore, choose $V^{i}$ small enough such that for every $q \in V^{i},\left|\mathcal{R}_{q}^{\mathcal{M}}\right|^{2} \geq\left|\mathcal{R}_{p_{i}}^{\mathcal{M}}\right|^{2}-\varepsilon$. For $h$ small enough, denote $V_{h}^{i}=\pi_{h}^{-1}\left(V^{i}\right)$. Assuming $V^{i}$ is regular enough, there exists $h_{\varepsilon}$ (depending on the partition), such that For $h<h_{\varepsilon}$ we can choose disjoint balls $\left\{B_{h}\left(q_{h}^{i, j}\right)\right\}_{j=1}^{n_{h}^{i}}$ of radius $h$, centered at $q_{h}^{i, j} \in V^{i}$, such that $B_{h}\left(q_{h}^{i, j}\right) \subset V_{h}^{i}$ and

$$
\sum_{j=1}^{n_{h}} \operatorname{Vol}_{\mathfrak{g}}\left(B_{h}\left(q_{h}^{i, j}\right)\right) \geq c \operatorname{Vol}_{\mathfrak{g}}\left(V_{h}^{i}\right)
$$

for some universal constant $c>0$ independent of $\varepsilon, i, h$ and $\mathcal{S}$. Now, for a given $u_{h} \in W^{1,2}\left(\mathcal{S}_{h} ; \mathbb{R}^{n}\right)$, we have

$$
\begin{aligned}
E_{\mathcal{S}_{h}}\left[u_{h}\right] & =\frac{1}{\operatorname{Vol}_{\mathfrak{g}}\left(\mathcal{S}_{h}\right)} \sum_{i=1}^{m} \int_{V_{h}^{i}} \operatorname{dist}^{2}\left(d u_{h}, \mathrm{SO}(\mathfrak{g}, \mathfrak{e})\right) d \operatorname{Vol}_{\mathfrak{g}} \\
& \geq \frac{1}{\operatorname{Vol}_{\mathfrak{g}}\left(\mathcal{S}_{h}\right)} \sum_{i=1}^{m} \sum_{j=1}^{n_{h}^{i}} \int_{B_{h}\left(q_{h}^{i, j}\right)} \operatorname{dist}^{2}\left(d u_{h}, \mathrm{SO}(\mathfrak{g}, \mathfrak{e})\right) d \operatorname{Vol}_{\mathfrak{g}} \\
& =\frac{1}{\operatorname{Vol}_{\mathfrak{g}}\left(\mathcal{S}_{h}\right)} \sum_{i=1}^{m} \sum_{j=1}^{n_{h}^{i}} \operatorname{Vol}_{\mathfrak{g}}\left(B_{h}\left(q_{h}^{i, j}\right)\right) E_{B_{h}\left(q_{h}^{i, j}\right)}\left[u_{h}\right] \\
& \geq \frac{1}{\operatorname{Vol}_{\mathfrak{g}}\left(\mathcal{S}_{h}\right)} \sum_{i=1}^{m} \sum_{j=1}^{n_{h}^{i}} \operatorname{Vol}_{\mathfrak{g}}\left(B_{h}\left(q_{h}^{i, j}\right)\right) \inf E_{B_{h}\left(q_{h}^{i, j}\right)} .
\end{aligned}
$$

Using Theorem 1.1 we then have

$$
\begin{aligned}
E_{\mathcal{S}_{h}}\left[u_{h}\right] & \geq \frac{1}{\operatorname{Vol}_{\mathfrak{g}}\left(\mathcal{S}_{h}\right)} \sum_{i=1}^{m} \sum_{j=1}^{n_{h}^{i}} \operatorname{Vol}_{\mathfrak{g}}\left(B_{h}\left(q_{h}^{i, j}\right)\right) \inf E_{B_{h}\left(q_{h}^{i, j}\right)} \\
& \geq \frac{1}{\operatorname{Vol}_{\mathfrak{g}}\left(\mathcal{S}_{h}\right)} \sum_{i=1}^{m} \sum_{j=1}^{n_{h}^{i}} \operatorname{Vol}_{\mathfrak{g}}\left(B_{h}\left(q_{h}^{i, j}\right)\right)\left(h^{4}\left|\mathcal{R}_{q_{h}^{i, j}}^{\mathcal{M}}\right|^{2}+o\left(h^{4}\right)\right) \\
& \geq \frac{1}{\operatorname{Vol}_{\mathfrak{g}}\left(\mathcal{S}_{h}\right)} \sum_{i=1}^{m} \sum_{j=1}^{n_{h}^{i}} \operatorname{Vol}_{\mathfrak{g}}\left(B_{h}\left(q_{h}^{i, j}\right)\right)\left(h^{4}\left(\left|\mathcal{R}_{p_{i}}^{\mathcal{M}}\right|^{2}-\varepsilon\right)+o\left(h^{4}\right)\right) \\
& \geq \operatorname{ch}^{4} \frac{1}{\operatorname{Vol}_{\mathfrak{g}}\left(\mathcal{S}_{h}\right)} \sum_{i=1}^{m}\left(\left|\mathcal{R}_{p_{i}}^{\mathcal{M}}\right|^{2}-\varepsilon\right) \operatorname{Vol}_{\mathfrak{g}}\left(V_{h}^{i}\right)+o\left(h^{4}\right) \\
& =\operatorname{ch}^{4}\left(\frac{1}{\operatorname{Vol}_{\mathfrak{g}}\left(\mathcal{S}_{h}\right)} \sum_{i=1}^{m}\left|\mathcal{R}_{p_{i}}^{\mathcal{M}}\right|^{2} \operatorname{Vol}_{\mathfrak{g}}\left(V_{h}^{i}\right)-\varepsilon\right)+o\left(h^{4}\right)
\end{aligned}
$$


where we used Corollary 3.7 for $K=\mathcal{S}$ to take the $o\left(h^{4}\right)$ term uniformly with respect to $q_{h}^{i, j}$ in last line. Now, using the fact that $\operatorname{Vol}_{\mathfrak{g}}\left(V_{h}^{i}\right)=h^{n-k} \operatorname{Vol}_{\mathfrak{g} \mid s}\left(V^{i}\right)(1+o(1))$ and $\operatorname{Vol}_{\mathfrak{g}}\left(\mathcal{S}_{h}\right)=h^{n-k} \operatorname{Vol}_{\left.\mathfrak{g}\right|_{\mathcal{S}}}(\mathcal{S})(1+o(1))$, we have

$$
\begin{aligned}
E_{\mathcal{S}_{h}}\left[u_{h}\right] & \geq \operatorname{ch}^{4}\left(\frac{1}{\operatorname{Vol}_{\mathfrak{g}}\left(\mathcal{S}_{h}\right)} \sum_{i=1}^{m}\left|\mathcal{R}_{p_{i}}^{\mathcal{M}}\right|^{2} \operatorname{Vol}_{\mathfrak{g}}\left(V_{h}^{i}\right)-\varepsilon\right)+o\left(h^{4}\right) \\
& =c^{4}\left(\frac{1}{\operatorname{Vol}_{\left.\mathfrak{g}\right|_{\mathcal{S}}}(\mathcal{S})} \sum_{i=1}^{m}\left|\mathcal{R}_{p_{i}}^{\mathcal{M}}\right|^{2} \operatorname{Vol}_{\left.\mathfrak{g}\right|_{\mathcal{S}}}\left(V^{i}\right)-\varepsilon\right)+o\left(h^{4}\right) \\
& \geq \operatorname{ch}^{4}\left(f_{\mathcal{S}}\left|\mathcal{R}^{\mathcal{M}}\right|^{2} d \operatorname{Vol}_{\left.\mathfrak{g}\right|_{\mathcal{S}}}-2 \varepsilon\right)+o\left(h^{4}\right) .
\end{aligned}
$$

Taking the infimum over $u_{h}$, dividing by $h^{4}$ and taking the limit $h \rightarrow 0$, we then have

$$
\liminf \left(\inf h^{-4} E_{\mathcal{S}_{h}}\right) \geq c\left(f_{\mathcal{S}}\left|\mathcal{R}^{\mathcal{M}}\right|^{2} d \operatorname{Vol}_{\left.\mathfrak{g}\right|_{\mathcal{S}}}-2 \varepsilon\right) .
$$

Since $\varepsilon$ is arbitrary, the proof is complete.

\section{References}

[AAE $\left.{ }^{+} 12\right]$ H. Aharoni, Y. Abraham, R. Elbaum, E. Sharon, and R. Kupferman, Emergence of spontaneous twist and curvature in non-Euclidean rods: Application to Erodium plant cells, Phys. Rev. Lett. 108 (2012), 238106. 1,2, 3, 5

[AESK11] S. Armon, E. Efrati, E. Sharon, and R. Kupferman, Geometry and mechanics of chiral pod opening, Science 333 (2011), 1726-1730. 2

[AKM $\left.{ }^{+} 16\right]$ H. Aharoni, J. M. Kolinski, M. Moshe, I. Meirzada, and E. Sharon, Internal stresses lead to net forces and torques on extended elastic bodies, Phys. Rev. Lett. 117 (2016), 124101. 1,2, 4, 5, 6

[ALL17] V. Agostiniani, A. Lucantonio, and D. Lučić, Heterogeneous elastic plates with in-plane modulation of the target curvature and applications to thin gel sheets, preprint, 2017. 3

[BBS55] B.A. Bilby, R. Bullough, and E. Smith, Continuous distributions of dislocations: A new application of the methods of Non-Riemannian geometry, Proc. Roy. Soc. A 231 (1955), 263-273. 2

[BK14] P. Bella and R.V. Kohn, Metric-induced wrinkling of a thin elastic sheet, Journal of Nonlinear Science 24 (2014), no. 6, 1147-1176. 3

[BLS16] K. Bhattacharya, M. Lewicka, and M. Schäffner, Plates with incompatible prestrain, Arch. Rational Mech. Anal. 221 (2016), no. 1, 143-181. 1, 3, 4, 5

[BS56] B.A. Bilby and E. Smith, Continuous distributions of dislocations. III, Proc. Roy. Soc. Edin. A 236 (1956), 481-505. 2

[Che00] B.Y. Chen, Riemannian submanifolds: A survey, Handbook of Differential Geometry, edited by F. Dillen and L. Verstraelen 1 (2000), 187-418. 21

[Cia88] P. G. Ciarlet, Mathematical elasticity, volume 1: Three-dimensional elasticity, Elsevier, 1988. 4

[Cia05] An introduction to differential geometry with applications to elasticity, Springer Netherlands, 2005. 15

[Cia13] _ Linear and nonlinear functional analysis with applications, SIAM, 2013. 11, 14 
[COT17] S. Conti, H. Olbermann, and I. Tobasco, Symmetry breaking in indented elastic cones, Mathematical Models and Methods in Applied Sciences 27 (2017), no. 2, 291-321. 3

[CRS17] M. Cicalese, M. Ruf, and F. Solombrino, On global and local minimizers of prestrained thin elastic rods, Calculus of Variations and Partial Differential Equations 56 (2017), no. 4,115 . 4

[dC92] M.P. do Carmo, Riemannian geometry, Birkhäuser, 1992. 20

[ESK09a] E. Efrati, E. Sharon, and R. Kupferman, Buckling transition and boundary layer in non-Euclidean plates, PRE 80 (2009), 016602. 3

[ESK09b] Elastic theory of unconstrained non-Euclidean plates, Journal of the Mechanics and Physics of Solids 57 (2009), 762-775. 3

[ESK11] _ Hyperbolic non-Euclidean elastic strips and almost minimal surfaces, PRE 83 (2011), 046602. 3

[FJM02] G. Friesecke, R.D. James, and S. Müller, A theorem on geometric rigidity and the derivation of nonlinear plate theory from three dimensional elasticity, Comm. Pure Appl. Math. 55 (2002), 1461-1506. 3, 9

[FJM06] A hierarchy of plate models derived from nonlinear elasticity by $\Gamma$-convergence, Arch. Rat. Mech. Anal. 180 (2006), 183-236. 3

[GSD16] D. Grossman, E. Sharon, and H. Diamant, Elasticity and fluctuations of frustrated nanoribbons, Phys. Rev. Lett. 116 (2016), 258105. 2

[KES07] Y. Klein, E. Efrati, and E. Sharon, Shaping of elastic sheets by prescription of nonEuclidean metrics, Science 315 (2007), 1116 - 1120. 2

[KM14] R. Kupferman and C. Maor, A Riemannian approach to the membrane limit in nonEuclidean elasticity, Comm. Contemp. Math. 16 (2014), no. 5, 1350052. 4

[KMS] R. Kupferman, C. Maor, and A. Shachar, Asymptotic rigidity of Riemannian manifolds, https://arxiv.org/abs/1701.08892 2

[KO18] Robert V. Kohn and Ethan O'Brien, On the bending and twisting of rods with misfit, Journal of Elasticity 130 (2018), no. 1, 115-143. 4

[Kon55] K. Kondo, Geometry of elastic deformation and incompatibility, Memoirs of the Unifying Study of the Basic Problems in Engineering Science by Means of Geometry (K. Kondo, ed.), vol. 1, 1955, pp. 5-17. 2

[KS12] R. Kupferman and Y. Shamai, Incompatible elasticity and the immersion of non-flat Riemannian manifolds in euclidean space, Israel Journal of Mathematics 190 (2012), no. 1, 135-156. 2

[KS14] R. Kupferman and J.P. Solomon, A Riemannian approach to reduced plate, shell, and rod theories, Journal of Functional Analysis 266 (2014), 2989-3039. 3, 5, 6, 14, 15, 21

[LDR95] H. Le-Dret and A. Raoult, The nonlinear membrane model as a variational limit of nonlinear three-dimensional elasticity, Journal de Mathematiques Pures et Appliquees 74 (1995), 549-578. 4

[LDR96] - The membrane shell model in nonlinear elasticity: A variational asymptotic deriva-

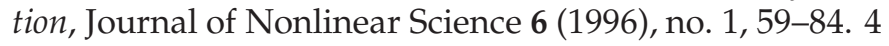

[LP11] M. Lewicka and M.R. Pakzad, Scaling laws for non-Euclidean plates and the $W^{2,2}$ isometric immersions of Riemannian metrics, ESAIM: Control, Optimisation and Calculus of Variations 17 (2011), 1158-1173. 2, 3

[LRR] M. Lewicka, A. Raoult, and D. Ricciotti, Plates with incompatible prestrain of higher order, To appear in Annales de l'Institut Henri Poincare (C) Non Linear Analysis. 1 , 3, 4, 5, 6

[Olb17] H. Olbermann, Energy scaling law for a single disclination in a thin elastic sheet, Arch. Rat. Mech. Anal. 224 (2017), no. 3, 985-1019. 3 
[OY09] A. Ozakin and A. Yavari, A geometric theory of thermal stresses, J. Math. Phys. 51 (2009), 032902. 2

[SRS07] E. Sharon, B. Roman, and H.L. Swinney, Geometrically driven wrinkling observed in free plastic sheets and leaves, PRE 75 (2007), 046211. 3

[Ten71] K. Tenenblat, On isometric immersions of Riemannian manifolds, Boletim da Soc. Bras. de Mat. 2 (1971), 23-36. 17, 21 